\title{
Achieving Synchronization in Arrays of Coupled Differential Systems with Time-Varying Couplings
}

\author{
Xinlei Yi, ${ }^{1}$ Wenlian Lu, ${ }^{1,2,3}$ and Tianping Chen ${ }^{1,4}$ \\ ${ }^{1}$ School of Mathematical Sciences, Fudan University, Shanghai 200433, China \\ ${ }^{2}$ Centre for Computational Systems Biology, Fudan University, Shanghai 200433, China \\ ${ }^{3}$ Centre for Scientific Computing, The University of Warwick, Coventry CV4 7AL, UK \\ ${ }^{4}$ School of Computer Science, Fudan University, Shanghai 200433, China
}

Correspondence should be addressed to Wenlian Lu; wenlian@fudan.edu.cn

Received 10 April 2013; Accepted 5 June 2013

Academic Editor: Zidong Wang

Copyright (C) 2013 Xinlei Yi et al. This is an open access article distributed under the Creative Commons Attribution License, which permits unrestricted use, distribution, and reproduction in any medium, provided the original work is properly cited.

\begin{abstract}
We study complete synchronization of the complex dynamical networks described by linearly coupled ordinary differential equation systems (LCODEs). Here, the coupling is timevarying in both network structure and reaction dynamics. Inspired by our previous paper (Lu et al. (2007-2008)), the extended Hajnal diameter is introduced and used to measure the synchronization in a general differential system. Then we find that the Hajnal diameter of the linear system induced by the time-varying coupling matrix and the largest Lyapunov exponent of the synchronized system play the key roles in synchronization analysis of LCODEs with identity inner coupling matrix. As an application, we obtain a general sufficient condition guaranteeing directed time-varying graph to reach consensus. Example with numerical simulation is provided to show the effectiveness of the theoretical results.
\end{abstract}

\section{Introduction}

Complex networks have widely been used in theoretical analysis of complex systems, such as Internet, World Wide Web, communication networks, and social networks. A complex dynamical network is a large set of interconnected nodes, where each node possesses a (nonlinear) dynamical system and the interaction between nodes is described as diffusion. Among them, linearly coupled ordinary differential equation systems (LCODEs) are a large class of dynamical systems with continuous time and state.

The LCODEs are usually formulated as follows:

$$
\dot{x}^{i}(t)=f\left(x^{i}(t)\right)+\sigma \sum_{j=1}^{m} l_{i j} B x^{j}(t), \quad i=1,2, \ldots, m,
$$

where $t \in \mathbb{R}^{+}=[0,+\infty)$ stands for the continuous time and $x^{i}(t) \in \mathbb{R}^{n}$ denotes the variable state vector of the $i$ th node, $f: \mathbb{R}^{n} \rightarrow \mathbb{R}^{n}$ represents the node dynamic of the uncoupled system, $\sigma \in \mathbb{R}^{+}=(0,+\infty)$ denotes coupling strength, $l_{i j} \geq 0$ with $i \neq j$ denotes the interaction between the two nodes, and $l_{i i}=-\sum_{j \neq i}^{m} l_{i j}, B \in \mathbb{R}^{n, n}$ denotes the inner coupling matrix. The LCODEs model is widely used to describe the model in nature and engineering. For example, the authors study spikeburst neural activity and the transitions to a synchronized state using a model of linearly coupled bursting neurons in [1]; the dynamics of linearly coupled Chua circuits are studied with application to image processing and many other cases in [2].

For decades, a large number of papers have focused on the dynamical behaviors of coupled systems [3-5], especially the synchronizing characteristics. The word "synchronization" comes from Greek; in this paper the concept of local complete synchronization (synchronization for simplicity) is considered (see Definition 3). For more details, we refer the readers to [6] and the references therein.

Synchronization of coupled systems have attracted a great deal of attention [7-9]. For instances, in [7], the authors considered the synchronization of a network of linearly coupled and not necessarily identical oscillators; in [8], the authors studied globally exponential synchronization for linearly coupled neural networks with time-varying delay and impulsive disturbances. Synchronization of networks with 
time-varying topologies was studied in [10-16]. For example, in [10], the authors proposed the global stability of total synchronization in networks with different topologies; in [16], the authors gave a result that the network will synchronize with the time-varying topology if the time-average is achieved sufficiently fast.

Synchronization of LCODEs has also been addressed in [17-19]. In [17], mathematical analysis was presented on the synchronization phenomena of LCODEs with a single coupling delay; in [18], based on geometrical analysis of the synchronization manifold, the authors proposed a novel approach to investigate the stability of the synchronization manifold of coupled oscillators; in [19], the authors proposed new conditions on synchronization of networks of linearly coupled dynamical systems with non-Lipschitz righthandsides. The great majority of research activities mentioned above all focused on static networks whose connectivity and coupling strengths are static. In many applications, the interaction between individuals may change dynamically. For example, communication links between agents may be unreliable due to disturbances and/or subject to communication range limitations.

In this paper, we consider synchronization of LCODEs with time-varying coupling. Similar to [17-19], time-varying coupling will be used to represent the interaction between individuals. In $[6,13]$, they showed that the Lyapunov exponents of the synchronized system and the Hajnal diameter of the variational equation play key roles in the analysis of the synchronization in the discrete-time dynamical networks. In this paper, we extend these results to the continuous-time dynamical network systems. Different from [11, 16], where synchronization of fast-switching systems was discussed, we focus on the framework of synchronization analysis with general temporal variation of network topologies. Additional contributions of this paper are that we explicitly show that (a) the largest projection Lyapunov exponent of a system is equal to the logarithm of the Hajnal diameter, and (b) the largest Lyapunov exponent of the transverse space is equal to the largest projection Lyapunov exponent under some proper conditions.

The paper is organized as follows: in Section 2, some necessary definitions, lemmas, and hypotheses are given; in Section 3, synchronization of generalized coupled differential systems is discussed; in Section 4, criteria for the synchronization of LCODEs are obtained; in Section 5, we obtain a sufficient condition ensuring directed time-varying graph reaching consensus; in Section 6, example with numerical simulation is provided to show the effectiveness of the theoretical results; the paper is concluded in Section 7.

Notions. $e_{k}^{n}=[0,0, \ldots, 0,1,0, \ldots, 0]^{\top} \in \mathbb{R}^{n}$ denotes the $n$ dimensional vector with all components zero except the $k$ th component $1, \mathbf{1}_{n}$ denotes the $n$-dimensional column vector with each component 1 ; for a set in some Euclidean space $U, \bar{U}$ denotes the closure of $U, U^{c}$ denotes the complementary set of $U$, and $A \backslash B=A \cap B^{c}$; for $u=\left[u_{1}, \ldots, u_{n}\right]^{\top} \in \mathbb{R}^{n},\|u\|$ denotes some vector norm, and for any matrix $A=\left(a_{i j}\right) \in \mathbb{R}^{n, m}$, $\|A\|$ denotes some matrix norm induced by vector norm, for example, $\|u\|_{1}=\sum_{i=1}^{n}\left|u_{i}\right|$ and $\|A\|_{1}=\max _{j} \sum_{i=1}^{n}\left|a_{i j}\right|$; for a matrix $A=\left(a_{i j}\right) \in \mathbb{R}^{n, m},|A|$ denotes a matrix with $|A|=\left(\left|a_{i j}\right|\right)$; for a real matrix $A, A^{\top}$ denotes its transpose and for a complex matrix $B, B^{*}$ denotes its conjugate transpose; for a set in some Euclidean space $W, \mathcal{O}(W, \delta)=\{x: \operatorname{dist}(x$, $W)<\delta\}$, where $\operatorname{dist}(x, W)=\inf _{y \in W}\|x-y\| ; \# J$ denotes the cardinality of set $J ;\lfloor z\rfloor$ denotes the floor function, that is, the largest integer not more than the real number $z ; \otimes$ denotes the Kronecker product; for a set in some Euclidean space $W$, $W^{m}$ denote the Cartesian product $W \times \cdots \times W$ ( $m$ times).

\section{Preliminaries}

In this section we will give some necessary definitions, lemmas, and hypotheses. Consider the following general coupled differential system:

$$
\dot{x}^{i}(t)=f^{i}\left(x^{1}(t), x^{2}(t), \ldots, x^{m}(t), t\right), \quad i=1,2, \ldots, m,
$$

with initial state $x\left(t_{0}\right)=\left[x^{1}\left(t_{0}\right)^{\top}, \ldots, x^{m}\left(t_{0}\right)^{\top}\right]^{\top} \in \mathbb{R}^{n m}$, where $t_{0} \in \mathbb{R}^{+}$denotes the initial time, $t \in \mathbb{R}^{+}$denotes the continuous time, and $x^{i}(t)=\left[x_{1}^{i}(t), \ldots, x_{n}^{i}(t)\right] \in \mathbb{R}^{n}$ denotes the variable state of the $i$ th node, $i=1,2, \ldots, m$.

For the functions $f^{i}: \mathbb{R}^{n m} \times \mathbb{R}^{+} \rightarrow \mathbb{R}^{n}, i=1,2, \ldots, m$, we make the following assumption.

Assumption 1. (a) There exists a function $f: \mathbb{R}^{n} \rightarrow \mathbb{R}^{n}$ such that $f^{i}(s, s, \ldots, s, t)=f(s)$ for all $i=1,2, \ldots, m, s \in \mathbb{R}^{n}$, and $t \geq 0$; (b) for any $t \geq 0, f^{i}(\cdot, t)$ is $C^{1}$-smooth for all $x=\left[x^{1^{\top}}, \ldots, x^{m \top}\right]^{\top} \in \mathbb{R}^{n m}$, and by $D F^{t}(x)=\left(\left(\partial f^{i} / \partial x^{j}\right)(x\right.$, t) $)_{i, j=1}^{m} \in \mathbb{R}^{n m, n m}$ denotes the Jacobian matrix of $F(x, t)=$ $\left[f^{i}(x, t)^{\top}, \ldots, f^{m}(x, t)^{\top}\right]^{\top}$ with respect to $x \in \mathbb{R}^{n m} ;(\mathrm{c})$ there exists a locally bounded function $\phi(x)$ such that $\left\|D F^{t}(x)\right\| \leq$ $\phi(x)$ for all $(x, t) \in \mathbb{R}^{n m} \times \mathbb{R}^{+} ;(\mathrm{d}) D F^{t}(x)$ is uniformly locally Lipschitz continuous: there exists a locally bounded function $K(x, y)$ such that

$$
\left\|D F^{t}(x)-D F^{t}(y)\right\| \leq K(x, y)\|x-y\|
$$

for all $t \geq 0$ and $x, y \in \mathbb{R}^{n m}$; (e) $f^{i}(x, t)$ and $D F^{t}(x)$ are both measurable for $t \geq 0$.

We say a function $g(y): \mathbb{R}^{q} \rightarrow \mathbb{R}^{p}$ is locally bounded if for any compact set $K \subset \mathbb{R}^{q}$, there exists $M>0$ such that $\|g(y)\| \leq M$ holds for all $y \in K$.

The first item of Assumption 1 ensures that the diagonal synchronization manifold

$$
\begin{array}{r}
\mathcal{S}=\left\{\left[x^{1 \top}, x^{2 \top}, \ldots, x^{m \top}\right]^{\top} \in \mathbb{R}^{n m}: x^{i \top}=x^{j \top},\right. \\
i, j=1,2, \ldots, m\}
\end{array}
$$

is an invariant manifold for (2).

If $x^{1}(t)=x^{2}(t)=\cdots=x^{m}(t)=s(t) \in \mathbb{R}^{n}$ is the synchronized state, then the synchronized state $s(t)$ satisfies

$$
\dot{s}(t)=f(s(t)) .
$$


Since $f(\cdot)$ is $C^{1}$-smooth, then $s(t)$ can be denoted by the corresponding continuous semiflow $s(t)=\vartheta^{(t)} s_{0}$ of the intrinsic system (5). For $\vartheta^{(t)}$, we make following assumption.

Assumption 2. The system (5) has an asymptotically stable attractor: there exists a compact set $A \subset R^{n}$ such that (a) $A$ is invariant through the system (5), that is, $\vartheta^{(t)} A \subset A$ for all $t \geq 0$; (b) there exists an open bounded neighborhood $U$ of $A$ such that $\bigcap_{t \geq 0} 9^{(t)} \bar{U}=A$; (c) $A$ is topologically transitive; that is, there exists $s_{0} \in A$ such that $\omega\left(s_{0}\right)$, the $\omega$ limit set of the trajectory $\vartheta^{(t)} s_{0}$, is equal to $A$ [3].

Definition 3. Local complete synchronization (synchronization for simplicity) is defined in the sense that the set

$$
\mathcal{S} \bigcap A^{m}=\left\{\left[x^{\top}, x^{\top}, \ldots, x^{\top}\right]^{\top} \in \mathbb{R}^{n m}: x^{\top} \in A\right\}
$$

is an asymptotically stable attractor in $\mathbb{R}^{n m}$. That is, for the coupled dynamical system (2), differences between components converge to zero if the initial states are picked sufficiently near $\delta \cap A^{m}$, that is, if the components are all close to the attractor $A$ and if their differences are sufficiently small.

Next we give some lemmas which will be used later, and the proofs can be seen in the appendix.

Lemma 4. Under Assumption 1, one has

$$
\sum_{j=1}^{m} \frac{\partial f^{i}}{\partial x^{j}}(\widehat{s}, t)=\frac{\partial f}{\partial s}(s)
$$

for all $s \in R^{n}$ and $t \geq 0$, where $\widehat{s}=\left[s^{\top}, s^{\top}, \ldots, s^{\top}\right]^{\top}$.

Lemma 5. Under Assumptions 1 and 2, there exists a compact neighborhood $W$ of $A$ such that $\vartheta^{(t)} W \subset \vartheta^{\left(t^{\prime}\right)} W$ for all $t \geq t^{\prime} \geq$ 0 and $\bigcap_{t \geq 0} \vartheta^{(t)} W=A$.

Let $\delta x(t)=\left[\delta x^{1}(t)^{\top}, \ldots, \delta x^{m}(t)^{\top}\right]^{\top} \in \mathbb{R}^{n m}$, where $\delta x^{i}(t)=$ $x^{i}(t)-s(t) \in \mathbb{R}^{n}$. We have the following variational equation near the synchronized state $s(t)$ :

$$
\delta \dot{x}^{i}(t)=\sum_{j=1}^{m} \frac{\partial f^{i}}{\partial x^{j}}(\widehat{s}(t), t) \delta x^{j}(t), \quad i=1,2, \ldots, m,
$$

or in matrix form:

$$
\delta \dot{x}(t)=D F^{t}(s(t)) \delta x(t),
$$

where $D F^{t}(s(t))$ denotes the Jacobin matrix $D F^{t}(\widehat{s}(t))$ for simplicity.

From [20], we can give the results on the existence, uniqueness, and continuous dependence of (2) and (9).

Lemma 6. Under Assumption 2, each of the differential equations (2) and (9) has a unique solution which is continuously dependent on the initial condition.
Thus, the solution of the linear system (9) can be written in matrix form.

Definition 7. Solution matrix $U\left(t, t_{0}, s_{0}\right)$ of the system (9) is defined as follows. Let $U\left(t, t_{0}, s_{0}\right)=\left[u^{1}\left(t, t_{0}, s_{0}\right), \ldots, u^{n m}(t\right.$, $\left.\left.t_{0}, s_{0}\right)\right]$, where $u^{k}\left(t, t_{0}, s_{0}\right)$ denotes the $k$ th column and is the solution of the following Cauchy problem:

$$
\begin{gathered}
\delta \dot{x}(t)=D F^{t}(s(t)) \delta x(t), \\
s\left(t_{0}\right)=s_{0}, \\
\delta x\left(t_{0}\right)=e_{k}^{n m} .
\end{gathered}
$$

Immediately, according to Lemma 6, we can conclude that the solution of the following Cauchy problem

$$
\begin{gathered}
\delta \dot{x}(t)=D F^{t}(s(t)) \delta x(t), \\
s\left(t_{0}\right)=s_{0}, \\
\delta x\left(t_{0}\right)=\delta x_{0}
\end{gathered}
$$

can be written as $\delta x(t)=U\left(t, t_{0}, s_{0}\right) \delta x_{0}$.

We define the time-varying Jacobin matrix $D F^{t}$ by the following way:

$$
\begin{aligned}
& D \mathscr{F}: \mathbb{R}^{+} \times R^{n} \longrightarrow 2^{\mathbb{R}^{n m, r m}}, \\
& \left(t_{0}, s_{0}\right) \longmapsto\left\{D F^{t}(s(t))\right\}_{t \geq t_{0}}
\end{aligned}
$$

with $s\left(t_{0}\right)=s_{0}$, where $2^{\mathbb{R}^{n m, n m}}$ is the collection of all the subsets of $\mathbb{R}^{n m, n m}$.

Definition 8 . For a time varying system denoted by $D \mathscr{F}$, we can define its Hajnal diameter of the variational system (9) as follows:

$$
\operatorname{diam}\left(D \mathscr{F}, s_{0}\right)=\varlimsup_{t \rightarrow \infty} \sup _{t_{0} \geq 0}\left\{\operatorname{diam}\left(U\left(t, t_{0}, s_{0}\right)\right)\right\}^{1 / t},
$$

where for a $\mathbb{R}^{n m, n m}$ matrix in block matrix form: $U=\left(U_{i j}\right)_{i, j=1}^{m}$ with $U_{i j} \in R^{n, n}$, its Hajnal diameter is defined as follows:

$$
\operatorname{diam}(U)=\max _{i, j}\left\|U_{i}-U_{j}\right\|,
$$

where $U_{i}=\left[U_{i 1}, U_{i 2}, \ldots, U_{i m}\right]$.

Lemma 9 (Grounwell-Beesack's inequality). If function $v(t)$ satisfies the following condition:

$$
v(t) \leq a(t)+b(t) \int_{0}^{t} v(\tau) d \tau,
$$

where $b(t) \geq 0$ and $a(t)$ are some measurable functions, then one has

$$
v(t) \leq a(t)+b(t) \int_{0}^{t} a(\tau) e^{\int_{\tau}^{t} b(\theta) d \theta} d \tau, \quad t \geq 0 .
$$

Based on Assumption 1, for the solution matrix $U$, we have the following lemma. 
Lemma 10. Under Assumption 1, one has the following:

(1) $\sum_{j=1}^{m} U_{i j}\left(t, t_{0}, s_{0}\right)=\breve{U}\left(t, t_{0}, s_{0}\right)$, where $\breve{U}\left(t, t_{0}, s_{0}\right)$ denotes the solution matrix of the following Cauchy problem:

$$
\begin{gathered}
\dot{u}=\frac{\partial f}{\partial s}(s(t)) u, \\
s\left(t_{0}\right)=s_{0} ;
\end{gathered}
$$

(2) for any given $t \geq 0$ and the compact set $W$ given in Lemma $5, U\left(t+t_{0}, t_{0}, s_{0}\right)$ is bounded for all $t_{0} \geq 0$ and $s_{0} \in W$ and equicontinuous with respect to $s_{0} \in W$.

Let $P=\left(P_{i j}\right)_{i, j=1}^{m}$ be a $\mathbb{R}^{n m, n m}$ matrix with $P_{i j} \in \mathbb{R}^{n, n}$ satisfying (a) $P_{i 1}=(1 / \sqrt{m}) P_{0}$ for some orthogonal matrix $P_{0} \in$ $\mathbb{R}^{n, n}$ and all $i=1,2, \ldots, m$; (b) $P$ is also an orthogonal matrix in $\mathbb{R}^{n m, n m}$. We also write $P$ and its inverse $P^{-1}=P^{\top}$ in the form

$$
P=\left[P_{1}, P_{2}\right], \quad P^{\top}=\left[\begin{array}{c}
P_{1}^{\top} \\
P_{2}^{\top}
\end{array}\right],
$$

where $P_{1}=(1 / \sqrt{m}) \mathbf{1}_{m} \otimes P_{0}$ and $P_{2} \in \mathbb{R}^{n m, n(m-1)}$. According to Lemma 10 , we have

$$
U\left(t, t_{0}, s_{0}\right) P_{1}=\frac{1}{\sqrt{m}} \mathbf{1}_{m} \otimes\left[\breve{U}\left(t, t_{0}, s_{0}\right) P_{0}\right] .
$$

Since $P_{2}^{\top} P_{1}=0$ which implies that each row of $P_{2}^{\top}$ is located in the subspace orthogonal to the subspace $\left\{\mathbf{1}_{m} \otimes \xi, \xi \in \mathbb{R}^{n}\right\}$, we can conclude that $P_{2}^{\top} U\left(t, t_{0}, s_{0}\right) P_{1}=0$. Then, we have

$$
P^{-1} U\left(t, t_{0}, s_{0}\right) P=\left[\begin{array}{cc}
P_{0}^{\top} \breve{U}\left(t, t_{0}, s_{0}\right) P_{0} & \alpha\left(t, t_{0}, s_{0}\right) \\
0 & \widetilde{U}\left(t, t_{0}, s_{0}\right)
\end{array}\right],
$$

where $\breve{U}\left(t, t_{0}, s_{0}\right)$ denotes the common row sum of $U\left(t, t_{0}\right.$, $\left.s_{0}\right)=\left(U_{i j}\right)_{i, j=1}^{m}$ as defined in Lemma 10, $\widetilde{U}\left(t, t_{0}, s_{0}\right)=P_{2}^{\top} U(t$, $\left.t_{0}, s_{0}\right) P_{2} \in \mathbb{R}^{n(m-1), n(m-1)}, \alpha\left(t, t_{0}, s_{0}\right) \in \mathbb{R}^{n, n(m-1)}$ denotes a matrix, and we omit its accurate expression. One can see that $\widetilde{U}\left(t, t_{0}, s_{0}\right)$ is the solution matrix of the following linear differential system.

Definition 11. We define the following linear differential system by the projection variational system of (9) along the directions $P_{2}$ :

$$
\begin{gathered}
\dot{\phi}=D_{P} F^{t}(s(t)) \phi, \\
s\left(t_{0}\right)=s_{0},
\end{gathered}
$$

where $D_{P} F^{t}(s(t))=P_{2}^{\top} D F^{t}(s(t)) P_{2}$.

Definition 12 . For any time varying variational system $D \mathscr{F}$ : $\mathbb{R}^{+} \times \mathbb{R}^{n} \rightarrow 2^{\mathbb{R}^{n m, n m}}$, we define the Lyapunov exponent of the variational system (9) as follows:

$$
\lambda\left(D \mathscr{F}, u, s_{0}\right)=\varlimsup_{t \rightarrow \infty} \sup _{t_{0} \geq 0} \frac{1}{t} \log \left\|U\left(t, t_{0}, s_{0}\right) u\right\|,
$$

where $u \in \mathbb{R}^{n m}$ and $s\left(t_{0}\right)=s_{0}$.
Similarly, we can define the projection Lyapunov exponents by the following projection time-varying variation:

$$
\begin{gathered}
D_{P} \mathscr{F}: \mathbb{R}^{+} \times \mathbb{R}^{n} \longrightarrow 2^{\mathbb{R}^{n(m-1), n(m-1)}}, \\
\left(t_{0}, s_{0}\right) \longmapsto\left\{D_{P} F^{t}(s(t))\right\}_{t \geq t_{0}},
\end{gathered}
$$

that is,

$$
\lambda\left(D_{P} \mathscr{F}, \tilde{u}, s_{0}\right)=\varlimsup_{t \rightarrow \infty} \sup _{t_{0} \geq 0} \frac{1}{t} \log \left\|\widetilde{U}\left(t, t_{0}, s_{0}\right) \tilde{u}\right\|,
$$

where $\tilde{u} \in \mathbb{R}^{n(m-1)}$ and $s\left(t_{0}\right)=s_{0}$. Let

$$
\lambda_{P}\left(D \mathscr{F}, s_{0}\right)=\max _{\tilde{u} \in \mathbb{R}^{n(m-1)}} \lambda\left(D_{P} \mathscr{F}, \tilde{u}, s_{0}\right) .
$$

Then, we have the following lemma.

Lemma 13. $\lambda_{P}\left(D \mathscr{F}, s_{0}\right)=\log \operatorname{diam}\left(D \mathscr{F}, s_{0}\right)$.

Remark 14. From Lemma 13, we can see that the largest projection Lyapunov exponent is independent of the choice of matrix $P$.

Consider the time-varying driven by some metric dynamical system $\operatorname{MDS}\left(\Omega, \mathscr{B}, \mathbb{P}, \varrho^{(t)}\right)$, where $\Omega$ is the compact state space, $\mathscr{B}$ is the $\sigma$-algebra, $\mathbb{P}$ is the probability measure, and $\varrho^{(t)}$ is a continuous semiflow. Then, the variational equation (9) is independent of the initial time $t_{0}$ and can be rewritten as follows:

$$
\begin{gathered}
\dot{\phi}=D F\left(s(t), \varrho^{(t)} \omega_{0}\right) \phi, \\
s(0)=s_{0} .
\end{gathered}
$$

In this case, we denote the solution matrix, the projection solution matrix, and the solution matrix on the synchronization space by $U\left(t, s_{0}, \omega_{0}\right), \widetilde{U}\left(t, s_{0}, \omega_{0}\right)$, and $\breve{U}\left(t, s_{0}, \omega_{0}\right)$, respectively. For simplicity, we write them as $U(t), \widetilde{U}(t)$, and $\breve{U}(t)$, respectively. Also, we write the Lyapunov exponents and the projection Lyapunov exponent as follows:

$$
\begin{aligned}
\lambda\left(D \mathscr{F}, u, s_{0}, \omega_{0}\right) & =\varlimsup_{t \rightarrow \infty} \frac{1}{t} \log \left\|U\left(t, s_{0}, \omega_{0}\right) u\right\|, \\
\lambda\left(D \mathscr{F}, s_{0}, \omega_{0}\right) & =\max _{u \in \mathbb{R}^{n m}} \lambda\left(D \mathscr{F}, u, s_{0}, \omega_{0}\right), \\
\lambda_{P}\left(D \mathscr{F}, u, s_{0}, \omega_{0}\right) & =\varlimsup_{t \rightarrow \infty} \frac{1}{t} \log \left\|\widetilde{U}\left(t, s_{0}, \omega_{0}\right) u\right\|, \\
\lambda_{P}\left(D \mathscr{F}, s_{0}, \omega_{0}\right) & =\max _{u \in \mathbb{R}^{n(m-1)}} \lambda\left(D_{P} \mathscr{F}, \widetilde{u}, s_{0}, \omega_{0}\right) .
\end{aligned}
$$

We add the following assumption.

Assumption 15. (a) $\varrho^{(t)}$ is a continuous semiflow; (b) $D F(s, \omega)$ is a continuous map for all $(s, \omega) \in \mathbb{R}^{n} \times \Omega$.

The following are involving linear differential systems. For more details, we refer the readers to [21]. For a continuous scalar function $u(t)$, we denote its Lyapunov exponent by

$$
\chi[u(t)]=\varlimsup_{t \rightarrow \infty} \frac{1}{t} \log |u(t)| .
$$


The following properties will be used later:

(1) $\chi\left[\prod_{k=1}^{n} c_{k} u_{k}(t)\right] \leq \sum_{k=1}^{n} \chi\left[u_{k}(t)\right]$, where $c_{k}, k=1,2$, $\ldots, n$, are constants;

(2) if $\lim _{t \rightarrow \infty}(1 / t) \log |u(t)|=\alpha$, which is finite, then $\chi[1 /(u(t))]=-\alpha ;$

(3) $\chi[u(t)+v(t)] \leq \max \{\chi[u(t)], \chi[v(t)]\}$;

(4) for a vector-value or matrix-value function $U(t)$, we define $\chi[U(t)]=\chi[\|U(t)\|]$.

For the following linear differential system:

$$
\dot{x}(t)=A(t) x(t),
$$

where $x(t) \in \mathbb{R}^{n}$, a transformation $x(t)=L(t) y(t)$ is said to be a Lyapunov transformation if $L(t)$ satisfies

(1) $L(t) \in C^{1}[0,+\infty)$;

(2) $L(t), \dot{L}(t), L^{-1}(t)$ are bounded for all $t \geq 0$.

It can be seen that the class of Lyapunov transformations forms a group and the linear system for $y(t)$ should be

$$
\dot{y}(t)=B(t) y(t),
$$

where $B(t)=L^{-1}(t) A(t) L(t)-L^{-1}(t) \dot{L}(t)$. Then, we say system (30) is a reducible system of system (29). We define the adjoint system of (29) by

$$
\dot{x}(t)=-A^{*}(t) x(t) .
$$

If letting $V(t)$ be the fundamental matrix of (29), then $\left[V^{-1}(t)\right]^{*}$ is the fundamental matrix of (31). Thus, we say the system (29) is a regular system if the adjoint systems (29) and (31) have convergent Lyapunov exponent series: $\left\{\alpha_{1}, \ldots, \alpha_{n}\right\}$ and $\left\{\beta_{1}, \ldots, \beta_{n}\right\}$, respectively, which satisfy $\alpha_{i}+\beta_{i}=0$ for $i=1,2, \ldots, n$, or its reducible system (30) is also regular.

Lemma 16. Suppose that Assumptions 1, 2, and 15 are satisfied. Let $\left\{\sigma_{1}, \sigma_{2}, \ldots, \sigma_{n}, \sigma_{n+1}, \ldots, \sigma_{n m}\right\}$ be the Lyapunov exponents of the variational system (26), where $\left\{\sigma_{1}, \ldots, \sigma_{n}\right\}$ correspond to the synchronization space and the remaining correspond to the transverse space. Let $\lambda_{T}\left(D \mathscr{F}, s_{0}, \omega_{0}\right)=\max _{i \geq n+1} \sigma_{i}$ and $\lambda_{S}\left(D \mathscr{F}, s_{0}, \omega_{0}\right)=\max _{1 \leq i \leq n} \sigma_{i}$. If $(a)$ the linear system (17) is a regular system, (b) $\left\|D F\left(s(t), \varrho^{(t)} \omega_{0}\right)\right\| \leq M$ for all $t \geq 0$, (c) $\lambda_{P}\left(D \mathscr{F}, s_{0}, \omega_{0}\right) \neq \lambda_{S}\left(D \mathscr{F}, s_{0}, \omega_{0}\right)$, then $\lambda_{T}\left(D \mathscr{F}, s_{0}, \omega_{0}\right)=$ $\lambda_{P}\left(D \mathscr{F}, s_{0}, \omega_{0}\right)$.

\section{General Synchronization Analysis}

In this section we provide a methodology based on the previous theoretical analysis to judge whether a general differential system can be synchronized or not.

Theorem 17. Suppose that $W \in \mathbb{R}^{n}$ is the compact subset given in Lemma 5, and Assumptions 1 and 2 are satisfied. If

$$
\sup _{s_{0} \in W} \operatorname{diam}\left(D \mathscr{F}, s_{0}\right)<1 \text {, }
$$

then the coupled system (2) is synchronized.
Proof. The main techniques of the proof come from $[3,6]$ with some modifications. Let $\vartheta^{(t)}$ be the semiflow of the uncoupled system (5). By the condition (32), there exist $d$ satisfying $\sup _{s_{0} \in W} \operatorname{diam}\left(D \mathscr{F}, s_{0}\right)<d<1$ and $T_{1} \geq 0$ such that $d^{T_{1}}<1 / 3$, and $r_{0}=\inf \left\{r>0, \mathcal{O}\left(\vartheta^{\left(T_{1}\right)} W, r\right) \subset W\right\}>0$. For each $s_{0} \in W$, there must exist $t\left(s_{0}\right) \geq T_{1}$ such that $\operatorname{diam}\left(U\left(t_{0}+t\left(s_{0}\right), t_{0}, s_{0}\right)\right)<d^{t\left(s_{0}\right)}$ for all $t_{0} \geq 0$. According to the equicontinuity of $U\left(t_{0}+t\left(s_{0}\right), t_{0}, s_{0}\right)$, there exists $\delta>0$ such that for any $s_{0}^{\prime} \in \mathcal{O}\left(s_{0}, \delta\right)$, $\operatorname{diam}\left(U\left(t_{0}+t\left(s_{0}\right), t_{0}, s_{0}^{\prime}\right)\right)<$ $d^{t\left(s_{0}\right)}$ for all $t_{0} \geq 0$. According to the compactness of $W$, there exists a finite positive number set $\mathscr{T}=\left\{t_{1}, t_{2}, \ldots, t_{v}\right\}$ with $t_{j} \geq T_{1}$ for all $j=1,2, \ldots, v$ such that for any $s_{0} \in W$, there exists $t_{j} \in \mathscr{T}$ such that $\operatorname{diam}\left(U\left(t_{0}+t_{j}, t_{0}, s_{0}\right)\right)<1 / 3$ for all $t_{0} \geq 0$. Let $x(t)$ be the collective states $\left\{x^{1}(t), \ldots, x^{m}(t)\right\}$ which is the solution of the coupled system (2) with initial condition $x^{i}\left(t_{0}\right)=x_{0}^{i}, i=1,2, \ldots, m$. And let $s(t)$ be the solution of the synchronization state equation (5) with initial condition $s\left(t_{0}\right)=\bar{x}_{0}=(1 / m) \sum_{j=1}^{m} x_{0}^{j} \in W$. Then, letting $\Delta x^{i}(t)=x^{i}(t)-s(t)$, we have

$$
\begin{aligned}
\Delta \dot{x}_{k}^{i}(t) & =f_{k}^{i}\left(x^{1}(t), \ldots, x^{m}(t), t\right)-f_{k}(s(t)) \\
& =\sum_{j=1}^{m} \sum_{l=1}^{n} \frac{\partial f_{k}^{i}}{\partial x_{l}^{j}}\left(\xi_{k l}^{i j}(t), t\right) \Delta x_{l}^{j}(t),
\end{aligned}
$$

where $\xi_{k l}^{i j}(t) \in \mathbb{R}^{m n}, i, j=1,2, \ldots, m, k, l=1,2 \ldots, n$, are obtained by the mean value principle of the differential functions. Letting $D F^{t}(\xi(t))=\left(\left(\partial f_{k}^{i} / \partial x_{l}^{i}\right)\left(\xi_{k l}^{i j}(t), t\right)\right)$, we can write the equations above in matrix form:

$$
\Delta \dot{x}(t)=D F^{t}(\xi(t)) \Delta x(t),
$$

and denote its solution matrix by $\widehat{U}\left(t+t_{0}, t_{0}, x_{0}\right)=\left(\widehat{U}_{i j}(t+\right.$ $\left.\left.t_{0}, t_{0}, x_{0}\right)\right)_{i, j=1}^{m}$. Then, for any $t>0$ there exists $K_{2}>0$ such that $\left\|D F^{t+t_{0}}\left(\xi\left(t+t_{0}\right)\right)\right\| \leq K_{2}$ for all $t \in \mathscr{T}$ and $t_{0} \geq 0$ according to the 3 th item of Assumption 1. Then, we have

$$
\begin{aligned}
& \Delta x_{k}^{i}\left(t+t_{0}\right) \\
& =x_{0}^{i}-\bar{x}_{0 k}+\int_{t_{0}}^{t+t_{0}} \sum_{j=1}^{m} \sum_{l=1}^{n} \frac{\partial f_{k}^{i}}{\partial x_{l}^{j}}\left(\xi_{k l}^{i j}(\tau), \tau\right) \Delta x_{l}^{j}(\tau) d \tau, \\
& \sum_{j=1}^{m} \sum_{k=1}^{n}\left\|\Delta x_{k}^{i}\left(t+t_{0}\right)\right\| \\
& \leq \sum_{j=1}^{m} \sum_{k=1}^{n}\left\|x_{0 k}^{i}-\bar{x}_{0 k}\right\|+K_{2} \int_{t_{0}}^{t+t_{0}} \sum_{j=1}^{m} \sum_{l=1}^{n}\left\|\Delta x_{l}^{j}(\tau)\right\| d \tau .
\end{aligned}
$$

By Lemma 9, we have

$$
\begin{aligned}
& \sum_{j=1}^{m} \sum_{l=1}^{n}\left\|\Delta x_{l}^{j}\left(t+t_{0}\right)\right\| \\
& \leq e^{K_{2} t} \sum_{j=1}^{m} \sum_{l=1}^{n}\left\|x_{0 l}^{j}-\bar{x}_{0 l}\right\| .
\end{aligned}
$$


Let

$$
W_{\alpha}=\left\{x=\left[x^{1^{\top}}, \ldots, x^{m \top}\right]^{\top}: \bar{x} \in W, \sum_{j=1}^{m}\left\|x^{j}-\bar{x}\right\| \leq \alpha\right\} .
$$

Picking $\alpha$ sufficiently small such that for each $x_{0} \in W_{\alpha}$, there exists $t \in \mathscr{T}$ such that $\sum_{j=1}^{m}\left\|\Delta x^{j}\left(t+t_{0}\right)\right\|<r_{0} / 2$ and $\operatorname{diam}(\widehat{U}(t+$ $\left.\left.t_{0}, t_{0}, x_{0}\right)\right)<1 / 2$ for all $t_{0} \geq 0$.

Thus, we are to prove synchronization step by step.

For any $x_{0} \in W_{\alpha}$, there exists $t^{\prime}=t\left(x_{0}\right) \in \mathscr{T}$ such that

$$
\begin{aligned}
& \left\|x^{i}\left(t^{\prime}+t_{0}\right)-x^{j}\left(t^{\prime}+t_{0}\right)\right\| \\
& =\left\|\Delta x^{i}\left(t^{\prime}+t_{0}\right)-\Delta x^{j}\left(t^{\prime}+t_{0}\right)\right\| \\
& \leq \sum_{k=1}^{m}\left\|\widehat{U}_{i k}\left(t^{\prime}+t_{0}, t_{0}, x_{0}\right)-\widehat{U}_{j k}\left(t^{\prime}+t_{0}, t_{0}, x_{0}\right)\right\|\left\|\Delta x_{0}^{k}\right\| \\
& \leq \operatorname{diam}\left(\widehat{U}\left(t^{\prime}+t_{0}, t_{0}, x_{0}\right)\right) \max _{i, j}\left\|x_{0}^{i}-x_{0}^{j}\right\| \\
& \leq \frac{1}{2} \max _{i, j}\left\|x_{0}^{i}-x_{0}^{j}\right\| .
\end{aligned}
$$

Therefore, we have $\max _{i, j}\left\|x^{i}\left(t^{\prime}+t_{0}\right)-x^{j}\left(t^{\prime}+t_{0}\right)\right\| \leq(1 /$ 2) $\max _{i, j}\left\|x_{0}^{i}-x_{0}^{j}\right\|$, which implies that $\bar{x}\left(t^{\prime}+t_{0}\right) \in W$ and $x\left(t^{\prime}+\right.$ $\left.t_{0}\right) \in W_{\alpha / 2}$.

Then, reinitiated with time $t^{\prime}+t_{0}$ and condition $x\left(t^{\prime}+\right.$ $\left.t_{0}\right)$, continuing with the phase above, we can obtain that $\lim _{t \rightarrow \infty} \max _{i, j}\left\|x^{i}(t)-x^{j}(t)\right\|=0$. Namely, the coupled system (2) is synchronized. Furthermore, from the proof, we can conclude that the convergence is exponential with rate $O\left(\delta^{t}\right)$ where $\delta=\sup _{s_{0} \in W} \operatorname{diam}\left(D \mathscr{F}^{t}, s_{0}\right)$, and uniform with respect to $t_{0} \geq 0$ and $x_{0} \in W_{\alpha}$. This completes the proof.

Remark 18. According to Assumption 2 that attractor $A$ is asymptotically stable and the properties of the compact neighbor $W$ given in Lemma 5, we can conclude that the quantity

$$
\sup _{s_{0} \in W} \operatorname{diam}\left(D \mathscr{F}, s_{0}\right)
$$

is independent on the choice of $W$.

If the timevariation is driven by some $\operatorname{MDS}(\Omega, \mathscr{B}, \mathbb{P} P$, $\left.\varrho^{(t)}\right)$ and there exists a metric dynamical system $\{W \times$ $\left.\Omega, \mathbf{F}, \mathbf{P}, \pi^{(t)}\right\}$, where $\mathbf{F}$ is the product $\sigma$-algebra on $W \times \Omega, \mathbf{P}$ is the probability measure, and $\pi^{(t)}\left(s_{0}, \omega\right)=\left(\theta^{(t)} s_{0}, \varrho^{(t)} \omega\right)$. From Theorem 17, we have the following.

Corollary 19. Suppose that the conditions in Lemma 16 are satisfied, $W \times \Omega$ is compact in the topology defined in this $M D S$, the semiflow $\pi^{(t)}$ is continuous, and on $W \times \Omega$ the Jacobian matrix $D F\left(\theta^{(t)} s_{0}, \varrho^{(t)} \omega\right)$ is continuous. Let $\left\{\sigma_{i}\right\}_{i=1}^{n m}$ be the Lyapunov exponents of this MDS with multiplicity and $\left\{\sigma_{i}\right\}_{i=1}^{n}$ correspond to the synchronization space. If

$$
\sup _{\mathbf{P} \in \operatorname{Erg}_{\pi}(W \times \Omega)} \sup _{i \geq n+1} \sigma_{i}<0,
$$

where $\operatorname{Erg}_{\pi}(W \times \Omega)$ denotes the ergodic probability measure set supported in the MDS $\left\{W \times \Omega, \mathbf{F}, \mathbf{P}, \pi^{(t)}\right\}$, then the coupled system (2) is synchronized.

\section{Synchronization of LCODEs with Identity Inner Coupling Matrix and Time-Varying Couplings}

In this section we study synchronization in linearly coupled ordinary differential equation systems (LCODEs) with timevarying couplings. Considering the following LCODEs with identity inner coupling matrix:

$$
\dot{x}^{i}(t)=f\left(x^{i}(t)\right)+\sigma \sum_{j=1}^{m} l_{i j}(t) x^{j}(t), \quad i=1,2, \ldots, m,
$$

where $x^{i}(t) \in \mathbb{R}^{n}$ denotes the state variable of the $i$ th node, $f(\cdot): \mathbb{R}^{n} \rightarrow \mathbb{R}^{n}$ is a differential map, $\sigma \in \mathbb{R}^{+}$denotes coupling strength, and $l_{i j}(t)$ denotes the coupling coefficient from node $j$ to $i$ at time $t$, for all $i \neq j$, which are supposed to satisfy the following assumption. Here, we highlight that the inner coupling matrix is the identity matrix.

Assumption 20. (a) $l_{i j}(t) \geq 0, i \neq j$ are measurable and $l_{i i}(t)=$ - $\sum_{j=1, j \neq i}^{m} l_{i j}(t)$; (b) there exists $M_{1}>0$ such that $\left|l_{i j}(t)\right| \leq M_{1}$ for all $i, j=1,2, \ldots, m$.

Similarly, we can define the Hajnal diameter of the following linear system:

$$
\dot{u}(t)=\sigma L(t) u(t) .
$$

Let $V(t)=\left(v_{i j}(t)\right)_{i, j=1}^{m}$ be the fundamental solution matrix of the system (42). Then, its solution matrix can be written as $V\left(t, t_{0}\right)=V(t) V\left(t_{0}\right)^{-1}$. Thus, the Hajnal diameter of the system (42) can be defined as follows:

$$
\operatorname{diam}(\mathscr{L})=\varlimsup_{t \rightarrow \infty} \sup _{t_{0} \geq 0}\left\{\operatorname{diam}\left(V\left(t, t_{0}\right)\right)\right\}^{1 / t} .
$$

By Theorem 17, we have the following theorem.

Theorem 21. Suppose Assumptions 1, 2, and 20 are satisfied. Let $\mu$ be the largest Lyapunov exponent of the synchronized system $\dot{s}(t)=f(s(t))$, that is,

$$
\mu=\sup _{s_{0} \in W} \max _{u \in \mathbb{R}^{n}} \lambda\left(D f, u, s_{0}\right) .
$$

If $\log (\operatorname{diam}(\mathscr{L}))+\mu<0$, then the LCODEs (41) is synchronized.

Proof. Considering the variational equation of (41):

$$
\delta \dot{x}(t)=\left\{I_{m} \otimes D f(s(t))+\sigma L(t) \otimes I_{n}\right\} \delta x(t) .
$$


Let $\breve{U}\left(t, t_{0}, s_{0}\right)$ be the solution matrix of the synchronized state system (17) and $V\left(t, t_{0}\right)=\left(v_{i j}\left(t, t_{0}\right)\right)_{i, j=1}^{m}$ be the solution matrix of the linear system (42). We can see that $V\left(t, t_{0}\right) \otimes$ $\breve{U}\left(t, t_{0}, s_{0}\right)$ is the solution matrix of the variational system (45). Then,

$$
\begin{gathered}
\operatorname{diam}\left(V\left(t+t_{0}, t_{0}\right) \otimes \breve{U}\left(t+t_{0}, t_{0}, s_{0}\right)\right) \\
=\max _{i, j=1, \ldots, m_{k=1}} \sum_{i k}^{m}\left|v_{i k}\left(t+t_{0}, t_{0}\right)-v_{j k}\left(t+t_{0}, t_{0}\right)\right| \\
\times\left\|\breve{U}\left(t+t_{0}, t_{0}, s_{0}\right)\right\| \\
=\operatorname{diam}\left(V\left(t+t_{0}, t_{0}\right)\right)\left\|\breve{U}\left(t+t_{0}, t_{0}, s_{0}\right)\right\| .
\end{gathered}
$$

This implies that the Hajnal diameter of the variational system (45) is less than $e^{\mu} \operatorname{diam}(\mathscr{L})$. This completes the proof according to Theorem 17.

For the linear system (42), we firstly have the following lemma.

Lemma 22 (see [22]). $V\left(t, t_{0}\right)$ is a stochastic matrix.

From Lemmas 13 and 16, we have the following corollary.

Corollary 23. $\log \operatorname{diam}(\mathscr{L})=\lambda_{P}(\mathscr{L})$, where $\lambda_{P}(\mathscr{L})$ denotes the largest one of all the projection Lyapunov exponents of system (41). Moreover, if the conditions in Lemma 16 are satisfied, then $\log \operatorname{diam}(\mathscr{L})=\lambda_{T}(\mathscr{L})$, where $\lambda_{T}(\mathscr{L})$ denotes the largest one of all the Lyapunov exponents corresponding to the transverse space, that is, the space orthogonal to the synchronization space.

If $L(t)$ is periodic, we have the following.

Corollary 24. Suppose that $L(t)$ is periodic. Let $\varsigma_{i}, i=$ $1,2, \ldots, m$, are the Floquet multipliers of the linear system (42). Then, there exists one multiplier denoted by $\varsigma_{1}=1$ and $\operatorname{diam}(\mathscr{L})=\max _{\mathrm{i} \geq 2} \varsigma_{i}$.

If $L(t)=L\left(\varrho^{(t)} \omega\right)$ is driven by some $\operatorname{MDS}\left(\Omega, \mathscr{B}, P, \varrho^{(t)}\right)$, from Corollaries 19 and 23, we have the following corollary.

Corollary 25. Suppose $L(\omega)$ is continuous on $\Omega$ and conditions in Lemma 16 are satisfied. Let $\mu=\sup _{s_{0} \in W} \max _{u \in \mathbb{R}^{n}} \lambda(D f$,

$\left.u, s_{0}\right), \varsigma_{i}, i=1,2, \ldots, m$, be the Lyapunov exponents of the linear system (42) with $\varsigma_{1}=0$, and $\varsigma=\sup _{P \in \operatorname{Erf}_{\theta}(\Omega)} \max _{i \geq 2} \varsigma_{i}$. If $\mu+\varsigma<0$, then the coupled system (41) is synchronized.

Let $\mathscr{I}$ be the set consisting of all compact time intervals in $[0,+\infty)$ and $\mathscr{G}$ be the the set consisting of all graph with vertex set $\mathcal{N}=\{1,2, \ldots, m\}$.

Define

$$
\begin{gathered}
G: \mathscr{I} \times R^{+} \longrightarrow \mathscr{G}, \\
\left(I=\left[t_{1}, t_{2}\right], \delta\right) \longmapsto G(I, \delta),
\end{gathered}
$$

where $G(I, \delta)=\{\mathcal{N}, \mathscr{E}\}$ is a graph with vertex set $\mathcal{N}$ and its edge set $\mathscr{E}$ is defined as follows: there exists an edge from vertex $j$ to vertex $i$ if and only if $\int_{t_{1}}^{t_{2}} l_{i j}(\tau) d \tau>\delta$. Namely, we say that there is a $\delta$-edge from vertex $j$ to $i$ across $I=\left[t_{1}, t_{2}\right]$.

Definition 26. We say that the LCODEs (41) has a $\delta$-spanning tree across the time interval $I$ if the corresponding graph $G(I, \delta)$ has a spanning tree.

$$
\begin{aligned}
& \text { For a stochastic matrix } V=\left(v_{i j}\right)_{i, j=1}^{m} \text {, let } \\
& \qquad \eta(V)=\min _{i, j}\left\|v_{i} \wedge v_{j}\right\|_{1},
\end{aligned}
$$

where $v_{i}=\left[v_{i 1}, \ldots, v_{i m}\right], i=1,2, \ldots, m$, and $v_{i} \wedge v_{j}=$ $\left[\min \left\{v_{i 1}, v_{j 1}\right\}, \ldots, \min \left\{v_{i m}, v_{j m}\right\}\right]^{\top}$. Then, we can also define that $V$ is $\delta$-scrambling if $\eta(V)>\delta$.

Theorem 27. Suppose Assumption 20 is satisfied. $\operatorname{diam}(\mathscr{L})<$ 1 if and only if there exist $\delta>0$ and $T>0$ such that the LCODEs (41) has a $\delta$-spanning tree across any $T$-length time interval.

Remark 28. Different from [16], we do not need to assume that $L(t)$ has zero column sums and the timeaverage is achieved sufficiently fast.

Before proving this theorem, we need the following lemma.

Lemma 29. If the LCODEs (41) has a $\delta$-spanning tree across any $T$-length time interval, then there exist $\delta_{1}>0$ and $T_{1}>$ 0 such that $V\left(t, t_{0}\right)$ is $\delta_{1}$-scrambling for any $T_{1}$-length time interval.

Proof of Theorem 27. Sufficiency. From Lemma 29, we can conclude that there exist $\delta_{1}>0, \delta^{\prime}>0$, and $T_{1}>0$ such that $V\left(t, t_{0}\right)$ is $\delta_{1}$-scrambling across any $T_{1}$-length time

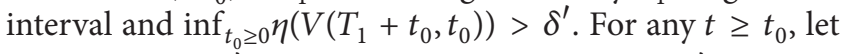
$t-t_{0}=p T_{1}+T^{\prime}$, where $p$ is an integer and $0 \leq T^{\prime}<T_{1}$ and $t_{l}=t_{0}+l T_{1}, 0 \leq l \leq p$. Then, we have

$$
\begin{aligned}
\operatorname{diam}\left(V\left(t, t_{0}\right)\right) & =\operatorname{diam}\left(V\left(t, t_{p}\right) \prod_{l=1}^{p} V\left(t_{l}, t_{l-1}\right)\right) \\
& \leq \operatorname{diam}\left(\prod_{l=1}^{p} V\left(t_{l}, t_{l-1}\right)\right) \\
& \leq 2 \prod_{l=1}^{p}\left(1-\eta\left(V\left(t_{l}, t_{l-1}\right)\right)\right) \\
& \leq 2\left(1-\delta^{\prime}\right)^{\left\lfloor\left(t-t_{0}\right) / T_{1}\right\rfloor} .
\end{aligned}
$$

For the first inequality, we use the results in $[23,24]$. This implies $\operatorname{diam}(\mathscr{L}) \leq\left(1-\delta^{\prime}\right)^{1 / T_{1}}<1$.

Necessity. Suppose that for any $T \geq 0$ and $\delta>0$, there exists $t_{0}=t_{0}(T, \delta), \int_{t_{0}}^{T+t_{0}} L(\tau) d \tau$ does not have a $\delta$-spanning tree. According to the condition, there exist $1>d>\operatorname{diam}(\mathscr{L})$, 
$\epsilon>0$, and $T^{\prime}>0$ such that $\operatorname{diam}\left(V\left(t+t_{0}\right)\right)<d^{t}$ for all $t_{0} \geq 0$ and $t \geq T^{\prime}$ and $d^{T^{\prime}}<1-\epsilon$. Thus, picking $T>T^{\prime}$, $\delta=m^{-3} e^{-M_{1} m T} \epsilon / 2, t_{1}=t_{0}(T, \delta)$, and $L^{\prime}=\left(l_{i j}^{\prime}\right)_{i, j=1}^{m}=$ $\left(\int_{T}^{T+t_{1}} l_{i j}(\tau) d \tau\right)_{i, j=1}^{m}$, there exist two vertex set $J_{1}$ and $J_{2}$ such that $l_{i j}^{\prime} \leq \delta$ if $i \in J_{1}$ and $j \notin J_{1}$, or $i \in J_{2}$ and $j \notin J_{2}$. For each $i \in J_{1}$ and $j \notin J_{1}$, we have

$$
\begin{aligned}
\dot{v}_{i j}(t)= & l_{i i}(t) v_{i j}(t)+\sum_{k \in J_{1}}^{k \neq i} l_{i k}(t) v_{k j}(t) \\
& +\sum_{k \notin J_{1}} l_{i k}(t) v_{k j}(t) \\
\leq & M_{1} \sum_{k \in J_{1}}^{k \neq i} v_{k j}(t)+\sum_{k \notin J_{1}} l_{i k}(t) .
\end{aligned}
$$

Then,

$$
\begin{aligned}
\sum_{i \in J_{1}, j \notin J_{1}} \dot{v}_{i j}(t) & \leq M_{1} \sum_{i \in J_{1}, k \in J_{1}}^{k \neq i, j \notin J_{1}} v_{k j}(t)+\sum_{i \in J_{1}, k \notin J_{1}}^{j \notin J_{1}} l_{i k}(t) \\
& =M_{1}\left(\# J_{1}-1\right) \sum_{k \in J_{1}}^{j \notin J_{1}} v_{k j}(t)+\left(m-\# J_{1}\right) \sum_{i \in J_{1}}^{k \notin J_{1}} l_{i k}(t) .
\end{aligned}
$$

Let $v(t)=\sum_{i \in J_{1}, j \notin J_{1}} v_{i j}(t)$. According to Lemma 9, we have

$$
\begin{aligned}
v\left(T+t_{1}\right) & \leq e^{M_{1}\left(\# J_{1}-1\right) T}\left(m-\# J_{1}\right) \int_{t_{1}}^{T+t_{1}} \sum_{i \in J_{1}}^{j \notin J_{1}} l_{i j}(\tau) d \tau \\
& \leq\left(m-\# J_{1}\right) e^{M_{1}\left(\# J_{1}-1\right) T} \# J_{1}\left(m-\# J_{1}\right) \delta \\
& \leq m^{3} e^{m M_{1} T} \delta \leq \frac{\epsilon}{2} .
\end{aligned}
$$

Similarly, we can conclude that $\sum_{i \in J_{l}, j \notin J_{l}} v_{i j}\left(T+t_{1}\right) \leq \epsilon / 2$ for all $l=1,2$. Without loss of generality, we suppose $J_{1}=$ $\{1,2, \ldots, p\}$ and $J_{2}=\{p+1, p+2, \ldots, p+q\}$, where $p$ and $q$ are integers with $p+q \leq m$. Then, we can write $V\left(T+t_{1}, t_{1}\right)$ in the following matrix form:

$$
V\left(T+t_{1}, t_{1}\right)=\left[\begin{array}{lll}
X_{11} & X_{12} & X_{13} \\
X_{21} & X_{22} & X_{23} \\
X_{31} & X_{32} & X_{33}
\end{array}\right],
$$

where $X_{11} \in \mathbb{R}^{p, p}$ and $X_{22} \in R^{q, q}$ correspond to the vertex subset $J_{1}$ and $J_{2}$, respectively. Immediately, we have $\left\|X_{12}\right\|_{\infty}+$ $\left\|X_{13}\right\|_{\infty}+\left\|X_{21}\right\|_{\infty}+\left\|X_{23}\right\|_{\infty} \leq \epsilon$. Let $v=\left[\begin{array}{c}1_{p} \\ 0 \\ 0\end{array}\right]$. We let

$$
V\left(t_{1}+T, t_{1}\right) v=\left[\begin{array}{ll}
X_{11} & \mathbf{1}_{p} \\
X_{21} & \mathbf{1}_{p} \\
X_{31} & \mathbf{1}_{p}
\end{array}\right] .
$$

Let $u=\left[\begin{array}{c}u^{1} \\ u^{2} \\ u^{3}\end{array}\right]=\left[u_{1}, \ldots, u_{m}\right]^{\top}$ with $u^{i}=\left[u_{1}^{i}, \ldots, u_{p_{i}}^{i}\right]^{\top}=$ $X_{i 1} \mathbf{1}_{p}$ and $p_{1}=p, p_{2}=q, p_{3}=m-p-q$. Then,

$$
\begin{aligned}
\max _{i, j}\left|u_{i}-u_{j}\right| & \geq \max _{k, l}\left|u_{k}^{1}-u_{j}^{2}\right| \\
& \geq 1-\left\|X_{12}\right\|_{\infty}-\left\|X_{13}\right\|_{\infty}-\left\|X_{21}\right\|_{\infty}-\left\|X_{23}\right\|_{\infty} \\
& \geq 1-\epsilon .
\end{aligned}
$$

Also,

$$
\max _{i, j}\left|u_{i}-u_{j}\right| \leq \operatorname{diam}\left(V\left(t_{1}+T, t_{1}\right)\right) \leq d^{T} .
$$

This implies $d^{T} \geq 1-\epsilon$ which leads contradiction with $d^{T}<$ $1-\epsilon$. Therefore, we can conclude the necessity.

\section{Consensus Analysis of Multiagent System with Directed Time-Varying Graphs}

If we let $n=1, f \equiv 0$, and $\sigma=1$ in system (41), then we have

$$
\dot{x}^{i}(t)=\sum_{j=1}^{m} l_{i j}(t) x^{j}(t), \quad i=1,2, \ldots, m .
$$

In this case, if Assumption 20 is satisfied, then the synchronization analysis of system (57) becomes another important research field named consensus problems.

Definition 30. We say the differential system (57) reaches consensus if for any $x\left(t_{0}\right) \in \mathbb{R}^{m},\left\|x^{i}(t)-x^{j}(t)\right\| \rightarrow 0$ as $t \rightarrow \infty$ for all $i, j \in \mathscr{N}$.

In graph view, the coefficients matrix of (57) $L(t)=$ $\left(l_{i j}(t)\right) \in \mathbb{R}^{m, m}$ is equal to the negative graph Laplacian associated with the digraph $G(t)$ at time $t$, where $G(t)=$ $(\mathscr{V}, \mathscr{E}(t), \mathscr{A}(t))$ is a weighted digraph (or directed graph) with $m$ vertices, the set of nodes $\mathscr{V}=\left\{v_{1}, \ldots, v_{m}\right\}$, set of edges $\mathscr{E}(t) \subseteq \mathscr{V} \times \mathscr{V}$, and the weighted adjacency matrix $\mathscr{A}(t)=$ $\left(a_{i j}(t)\right)$ with nonnegative adjacency elements $a_{i j}(t)$. An edge of $G(t)$ is denoted by $e_{i j}(t)=\left(v_{i}, v_{j}\right) \in \mathscr{E}(t)$ if there is a directed edge from vertex $i$ to vertex $j$ at time $t$. The adjacency elements associated with the edges of the graph are positive, that is, $e_{i j}(t) \in \mathscr{E}(t) \Leftrightarrow a_{i j}(t)>0$, for all $i, j \in \mathcal{N}$. It is assumed that $a_{i i}(t)=0$ for all $i \in \mathcal{N}$. The indegree and outdegree of node $v_{i}$ at time $t$ are, respectively, defined as follows:

$$
\operatorname{deg}_{\text {in }}\left(v_{i}(t)\right)=\sum_{j=1}^{N} a_{j i(t)}, \quad \operatorname{deg}_{\text {out }}\left(v_{i}(t)\right)=\sum_{j=1}^{N} a_{i j(t)} .
$$

The degree matrix of digraph $G(t)$ is defined as $D(t)=$ $\operatorname{diag}\left(\operatorname{deg}_{\text {out }}\left(v_{1}(t)\right), \ldots, \operatorname{deg}_{\text {out }}\left(v_{m}(t)\right)\right)$ at time $t$. The graph Laplacian associated with the digraph $G(t)$ at time $t$ is defined as

$$
-L(t)=\mathscr{L}(G(t))=D(t)-\mathscr{A}(t) .
$$


Let $G(I, \delta)$ defined as before. We say that the digraph $G(t)$ has a $\delta$-spanning tree across the time interval $I$ if $G(I, \delta)$ has a spanning.

Theorem 31. Suppose Assumption 20 is satisfied. The system (57) reaches consensus if and only if there exist $\delta>0$ and $T>0$ such that the corresponding digraph $G(t)$ has a $\delta$-spanning tree across any T-length time interval.

Proof. Since $f \equiv 0$, we have $\mu=0$ in Theorem 21. This completes the proof according to Theorems 27 and 21.

Remark 32. This theorem is a part of Theorem 17 in [25].

\section{Numerical Examples}

In this section, a numerical example is given to demonstrate the effectiveness of the presented results on synchronization of LCODEs with time-varying couplings. The Lyapunov exponents are computed numerically. By this way, we can verify the the synchronization criterion and analyze synchronization numerically. We use the Rössler system $[16,26]$ as the node dynamics

$$
\begin{gathered}
\dot{x}_{1}(t)=-x_{2}(t)-x_{3}(t), \\
\dot{x}_{2}(t)=x_{1}(t)+a x_{2}(t), \\
\dot{x}_{3}(t)=b+x_{3}(t)\left(x_{1}(t)-c\right),
\end{gathered}
$$

where $a=0.165, b=0.2$, and $c=10$. Figure 1 shows the dynamical behaviors of the Rössler system (60) with random initial value in $[0,1]$ that includes a chaotic attractor $[16,26]$.

The network with time-varying topology we used here is NW small-world network with a time-varying coupling, which was introduced as the blinking model in [11, 27]. The time-varying network model algorithm is presented as follows: we divide the time axis into intervals of length $\tau$, in each interval: (a) begin with the nearest neighbor coupled network consisting of $m$ nodes arranged in a ring, where each node $i$ is adjacent to its $2 k$-nearest neighbor nodes; (b) add a connection between each pair of nodes with probability $p$, which usually is a random number between $[0,0.1]$; for more details, we refer the readers to [11]. Figure 2 shows the timevarying structure of shortcut connections in the blinking model with $m=50$ and $k=3$.

In this example, the parameters are taken values as $m=$ $50, k=3, \tau=1$, and $p=0.04$. Then blinking small-world network can be generated with the coupling graph Laplacian $\mathscr{L}(G(t))=-L(t)$. The dynamical network system can be described as follows:

$$
\begin{array}{r}
\dot{x}_{1}^{i}(t)=-x_{2}^{i}(t)-x_{3}^{i}(t)+\sigma \sum_{j=1}^{m} l_{i j}(t) x_{1}^{j}(t), \\
\dot{x}_{2}^{i}(t)=x_{1}^{i}(t)+a x_{2}^{i}(t)+\sigma \sum_{j=1}^{m} l_{i j}(t) x_{2}^{j}(t), \\
\dot{x}_{3}^{i}(t)=b+x_{3}^{i}(t)\left(x_{1}^{i}(t)-c\right)+\sigma \sum_{j=1}^{m} l_{i j}(t) x_{3}^{j}(t), \\
i=1,2, \ldots, m .
\end{array}
$$

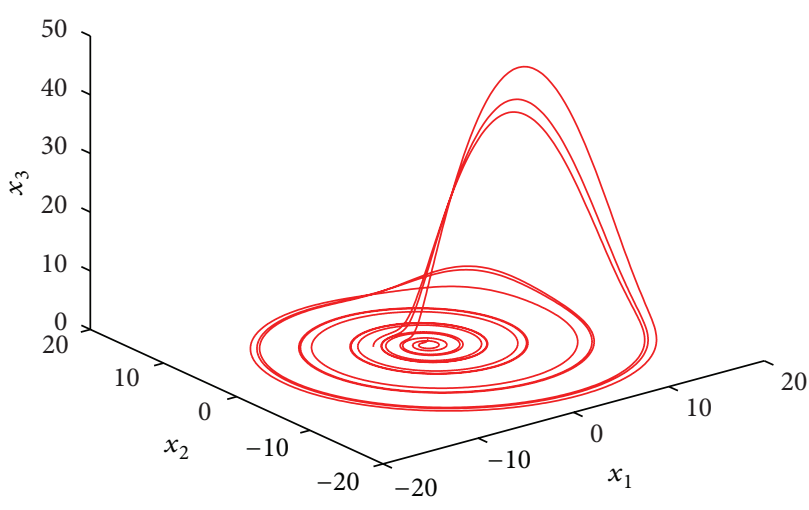

Figure 1: The dynamical behavior of the Rössler system (60) with $a=0.165, b=0.2$, and $c=10$.

Let $e(t)=\max _{1 \leq i<j \leq 50}\left\|x^{i}(t)-x^{j}(t)\right\|$ denotes the maximum distance between nodes at time $t$. Let $E=\int_{T}^{T+R} e(t) d t$, for some sufficiently large $T>0$ and $R>0$. Let $H=\mu+\varsigma$ defined in Corollary 25. As described in Corollary 25, two steps are needed for verification: (a) calculating the largest Lyapunov exponent of the uncoupled synchronized system (60), $\mu$ and (b) calculating the second largest Lyapunov exponent of the linear system (42). In detail, we use Wolf's method [28] to compute $\mu$ and the Jacobian method [29] to compute Lyapunov spectra of (42). More details can be found in [28-30]. Figure 3 shows convergence of the maximum distance between nodes during the topology evolution with a different coupling strength $\sigma$. It can be seen from Figure 3 that the dynamical network system (61) can be synchronized with $\sigma=0.4$ and $\sigma=0.5$.

We pick the time length 200. Let $T=190$ and $R=10$. And choose initial state randomly from the interval $[0,1]$. Figure 4 shows the variation of $E$ and $H$ with respect to the coupling strength $\sigma$. It can be seen that the parameter (coupling strength $\sigma$ ) region where $H$ is negative coincides with that of synchronization, that is, where $E$ is near zero. This verified the theoretical result (Corollary 25). In addition, we find that $\sigma \approx 0.38$ is the threshold for synchronizing the coupled systems in this case.

\section{Conclusions}

In this paper, we present a theoretical framework for synchronization analysis of general coupled differential dynamical systems. The extended Hajnal diameter is introduced to measure the synchronization. The coupling between nodes is timevarying in both network structure and reaction dynamics. Inspired by the approaches in $[6,13]$, we show that the Hajnal diameter of the linear system induced by the timevarying coupling matrix and the largest Lyapunov exponent of the synchronized system play the key roles in synchronization analysis of LCODEs. These results extend synchronization analysis of discrete-time network in [6] to continuoustime case. As an application, we obtain a very general sufficient condition ensuring directed time-varying graph reaching consensus, and the way we get this result is different from [25]. An example of numerical simulation is provided 


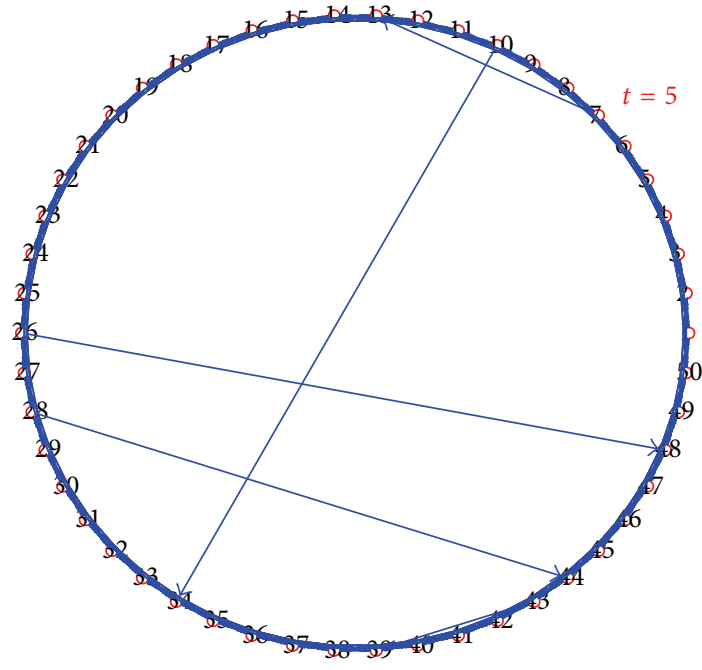

(a)

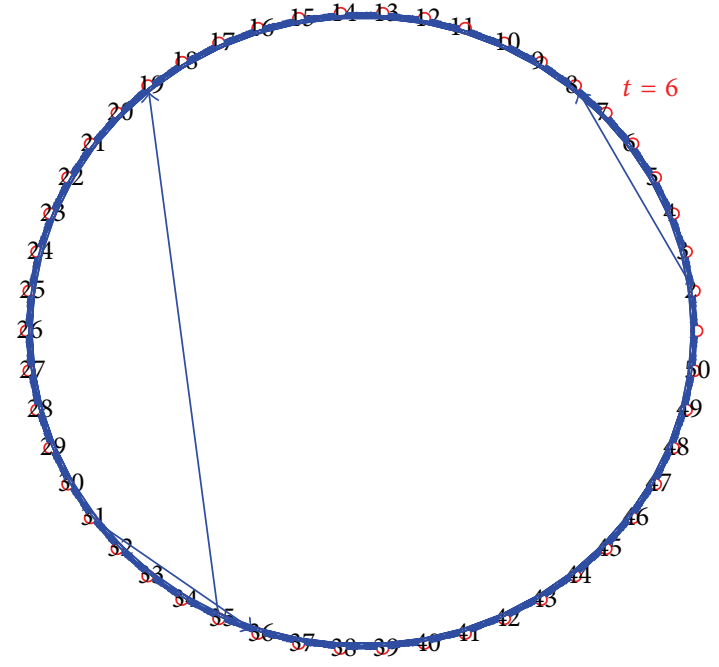

(b)

FIGURE 2: The blinking model of shortcuts connections. Probability of switchings $p=0.04$, the switching time step $\tau=1$.

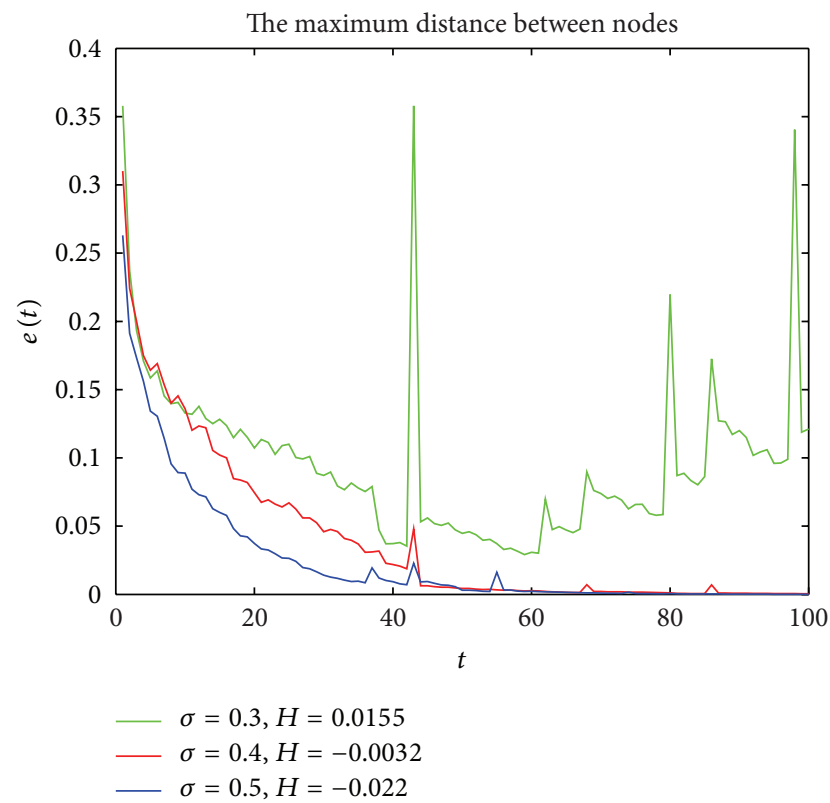

FIGURE 3: Convergence of the maximum distance between nodes with a different coupling strength $\sigma$.

to show the effectiveness the theoretical results. Additional contributions of this paper are that we explicitly show that the largest projection Lyapunov exponent, the Hajnal diameter, and the largest Lyapunov exponent of the transverse space are equal to each other in coupled differential systems (see Lemmas 13 and 16), which was proved in [6] for couple discrete-time systems.

\section{Appendix}

Proof of Lemma 5. Let $U$ be a bounded open neighborhood of $A$ satisfying $\bigcap_{t \geq 0} \vartheta^{(t)} \bar{U}=A$ and $U_{t}=\left\{x \in R^{n}: \vartheta^{(\tau)} x \in\right.$

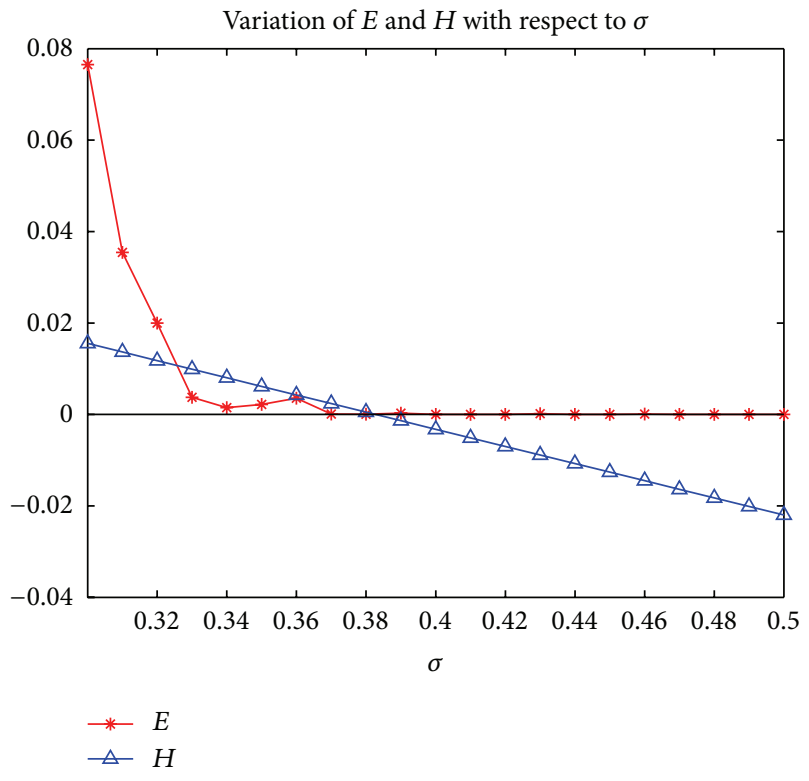

FIgURE 4: Variation of $e$ and $H$ with respect to $\sigma$ for the blinking topology.

$U, 0 \leq \tau \leq t\}$. This implies $U_{t} \supset U_{t^{\prime}}$ if $t^{\prime} \geq t \geq 0, U_{t}$ is an open set due to the continuity of the semiflow $\vartheta^{(t)}$, and $9^{(\delta)} U_{t} \subset U_{t-\delta}$ for all $t \geq \delta \geq 0$. Let $V=\bigcap_{t \geq 0} U_{t}$. We claim that there exists $t_{0} \geq 0$ such that $V=U_{t}$ for all $t \geq t_{0}$.

For any $\delta>0$, let $t_{n}=n \delta$ and $U_{n}=U_{t_{n}}$. We can conclude that $V=\bigcap_{n=1}^{\infty} U_{n}$. We will prove in the following that there exists $n_{0}$ such that $V=U_{n_{0}}$. Otherwise, there always exists $x_{n} \in U_{n} \backslash U_{n+1}$ for $n \geq 0$. Let $y_{n}=\vartheta^{\left(t_{n+1}\right)} x_{n}$. We have (i) $y_{n} \in \bigcap_{k=0}^{n} \vartheta^{\left(^{\left(t_{k}\right)}\right.} \bar{U}$ and (ii) $y_{n} \notin U$. For any limit point $\hat{y}$ of $y_{n}, \hat{y}$ can be either finite or infinite. For both cases, $\hat{y} \notin U$ which implies $\hat{y} \notin A$. However, the claim (i) implies that 
$\widehat{y} \in A$, which contradicts with the claim (ii). This completes the proof by letting $W=\bar{V}$.

Proof of Lemma 10. (a) For any initial condition with the form $\delta x_{0}=\mathbf{1}_{m} \otimes u_{0}$, the solution of (11) can be $U\left(t, t_{0}\right.$, $\left.s_{0}\right)\left(\mathbf{1}_{m} \otimes u_{0}\right)=\mathbf{1}_{m} \otimes \breve{U}\left(t, t_{0}, s_{0}\right) u_{0}$ according to Lemma 4 . This implies the first claim in this lemma.

(b) According to Lemma 5, there exists $K_{1}>0$ such that $s(t)$, the solution of (5), satisfies $\|s(t)\| \leq K_{1}$ for all $s_{0} \in W$ and $t \geq 0$. So, there exists $K>0$ such that $\left\|D F^{t}(s(t))\right\| \leq K$ according to the 3 th item of Assumption 1. Write the solution of (11) $\delta x(t)=U\left(t, t_{0}, s_{0}\right) \delta x_{0}$ as

$$
\delta x\left(t+t_{0}\right)=\delta x_{0}+\int_{t_{0}}^{t+t_{0}} D F^{\tau}(s(\tau)) \delta x(\tau) d \tau .
$$

Then,

$$
\begin{aligned}
& \left\|\delta x\left(t+t_{0}\right)\right\| \\
& \leq\left\|\delta x_{0}\right\|+\int_{t_{0}}^{t+t_{0}}\left\|D F^{\tau}(s(\tau))\right\|\|\delta x(\tau)\| d \tau \\
& \leq\left\|\delta x_{0}\right\|+K \int_{0}^{t}\left\|\delta x\left(\tau+t_{0}\right)\right\| d \tau .
\end{aligned}
$$

According to Lemma 9, we have $\left\|\delta x\left(t+t_{0}\right)\right\| \leq\left\|\delta x_{0}\right\|+$ $K \int_{0}^{t}\left\|\delta x_{0}\right\| e^{(t-\tau) K} d \tau=e^{K t}\left\|\delta x_{0}\right\|$. This implies that $\| U\left(t+t_{0}, t_{0}\right.$, $\left.s_{0}\right) \| \leq e^{K t}$ for all $s_{0} \in W$ and $t_{0} \geq 0$.

For any $s_{0}, s^{\prime}{ }_{0} \in W$, let $s(t)$ and $s^{\prime}(t)$ be the solution of the synchronized state equation (5) with initial condition $s\left(t_{0}\right)=$ $s_{0}$ and $s^{\prime}\left(t_{0}\right)=s_{0}^{\prime}$, respectively. We have

$$
\begin{aligned}
& s\left(t+t_{0}\right)-s^{\prime}\left(t+t_{0}\right) \\
& \quad=\int_{t_{0}}^{t+t_{0}}\left[f(s(\tau))-f\left(s^{\prime}(\tau)\right)\right] d \tau+s\left(t_{0}\right)-s^{\prime}\left(t_{0}\right), \\
& \left\|s\left(t+t_{0}\right)-s^{\prime}\left(t+t_{0}\right)\right\| \\
& \quad \leq\left\|s\left(t_{0}\right)-s^{\prime}\left(t_{0}\right)\right\|+K \int_{t_{0}}^{t+t_{0}}\left\|s(\tau)-s^{\prime}(\tau)\right\| d \tau .
\end{aligned}
$$

By Lemma 9, we have $\left\|s\left(t+t_{0}\right)-s^{\prime}\left(t+t_{0}\right)\right\| \leq e^{K t}\left\|s_{0}-s^{\prime}{ }_{0}\right\|$ for all $t_{0}, t \geq 0$ and $s_{0}, s^{\prime}{ }_{0} \in W$. Also, according to the 4 th item of Assumption 1, there must exist $K_{2}>0$ such that $\left\|D F^{t}(s(t))-D F^{t}\left(s^{\prime}(t)\right)\right\| \leq K_{2}\left\|s(t)-s^{\prime}(t)\right\|$ for all $t \geq 0$ and $s_{0}, s^{\prime}{ }_{0} \in W$. Then, let $\delta x(t)=U\left(t, t_{0}, s_{0}\right) \delta x_{0}, \delta y(t)=$ $U\left(t, t_{0}, s_{0}^{\prime}\right) \delta x_{0}$, and $v(t)=\delta x(t)-\delta y(t)$. We have

$$
\begin{aligned}
& v\left(t+t_{0}\right) \\
& =\int_{t_{0}}^{t+t_{0}}\left[D F^{\tau}(s(\tau)) \delta x(\tau)-D F^{\tau}\left(s^{\prime}(\tau)\right) \delta y(\tau)\right] d \tau \\
& =\int_{t_{0}}^{t+t_{0}}\left[D F^{\tau}(s(\tau))-D F^{\tau}\left(s^{\prime}(\tau)\right)\right] \delta x(\tau) d \tau \\
& \quad+\int_{t_{0}}^{t+t_{0}} D F^{\tau}\left(s^{\prime}(\tau)\right) v(\tau) d \tau,
\end{aligned}
$$

$$
\begin{aligned}
& \left\|v\left(t+t_{0}\right)\right\| \\
& \leq \int_{t_{0}}^{t+t_{0}}\left[\left\|D F^{\tau}(s(\tau))-D F^{\tau}\left(s^{\prime}(\tau)\right)\right\|\|\delta x(\tau)\|\right. \\
& \left.+\left\|D F^{\tau}\left(s^{\prime}(\tau)\right)\right\|\|v(\tau)\|\right] d \tau \\
& \leq K_{2} \int_{0}^{t} e^{2 K \tau} d \tau\left\|\delta x_{0}\right\|\left\|s_{0}-s^{\prime}{ }_{0}\right\| \\
& +K \int_{t_{0}}^{t_{0}+t}\|v(\tau)\| d \tau
\end{aligned}
$$

According to Lemma 9,

$$
\left\|v\left(t+t_{0}\right)\right\| \leq\left[\frac{K_{2}\left(e^{2 K t}-e^{K t}\right)}{K}\right]\left\|\delta x_{0}\right\|\left\|s_{0}-s^{\prime}{ }_{0}\right\| .
$$

This implies

$$
\begin{gathered}
\left\|U\left(t+t_{0}, t_{0}, s_{0}\right)-U\left(t+t_{0}, t_{0}, s^{\prime}{ }_{0}\right)\right\| \\
\leq\left[\frac{K_{2}\left(e^{2 K t}-e^{K t}\right)}{K}\right]\left\|s_{0}-s^{\prime}{ }_{0}\right\|
\end{gathered}
$$

for all $s_{0}, s_{0}^{\prime} \in W$. This completes the proof.

Proof of Lemma 13. We define the projection joint spectral radius as follows:

$$
\rho_{P}\left(D \mathscr{F}, s_{0}\right)=\varlimsup_{t \rightarrow \infty} \sup _{t_{0} \geq 0}\left\|\widetilde{U}\left(t, t_{0}, s_{0}\right)\right\|^{1 / t} .
$$

First, we will prove that $\operatorname{diam}\left(D \mathscr{F}, s_{0}\right)=\rho_{P}\left(D \mathscr{F}, s_{0}\right)$. For any $d>\rho_{P}\left(D \mathscr{F}, s_{0}\right)$, there exists $T \geq 0$ such that $\left\|\widetilde{U}\left(t+t_{0}, t_{0}, s_{0}\right)\right\| \leq$ $d^{t}$ for all $t_{0} \geq 0$ and $t \geq T$. This implies that

$$
\begin{aligned}
& \| P^{-1} U\left(t+t_{0}, t_{0}, s_{0}\right) P-\left[\begin{array}{c}
I_{n} \\
0 \\
\vdots \\
0
\end{array}\right] \\
& \times\left[P_{0}^{\top} \breve{U}\left(t+t_{0}, t_{0}, s_{0}\right) P_{0}, \alpha\left(t+t_{0}, t_{0}, s_{0}\right)\right] \\
& =\left\|\left[\begin{array}{lc}
0 & \widetilde{U}\left(t+t_{0}, t_{0}, s_{0}\right)
\end{array}\right]\right\| \leq C_{1} d^{t}
\end{aligned}
$$


for some $C_{1}>0$, all $t_{0} \geq 0$ and all $t \geq T$. Thus, there exist some $C_{2}>0$ and some matrix function $q(t) \in \mathbb{R}^{n, n m}$ such that

$$
\begin{aligned}
& \left\|U\left(t+t_{0}, t_{0}, s_{0}\right)-\mathbf{1}_{m} \otimes q(t)\right\| \\
& \left.=\| \begin{array}{c}
I_{n} \\
0 \\
\vdots \\
0
\end{array}\right] \\
& \quad \times\left[P_{0}^{\top} \breve{U}\left(t+t_{0}, t_{0}, s_{0}\right)-P P_{0}, \alpha\left(t+t_{0}, t_{0}, s_{0}\right)\right] P^{-1} v \| \\
& \leq C_{2} d^{t}
\end{aligned}
$$

for all $t_{0} \geq 0$ and $t \geq T$, where $q(t) \in \mathbb{R}^{n, n m}$ denotes a matrix, and we omit its accurate expression. So, we can conclude that $\operatorname{diam}\left(U\left(t+t_{0}, t_{0}, s_{0}\right)\right) \leq C_{3} d^{t}$ for some $C_{3}>0$, all $t_{0} \geq 0$, and $t \geq T$. This implies that $\operatorname{diam}\left(D \mathscr{F}, s_{0}\right) \leq d$, that is, $\operatorname{diam}\left(D \mathscr{F}, s_{0}\right) \leq \rho_{P}\left(D \mathscr{F}, s_{0}\right)$ due to the arbitrariness of $d \geq \rho_{P}\left(D \mathscr{F}, s_{0}\right)$. Conversely, for any $d>\operatorname{diam}\left(D \mathscr{F}, s_{0}\right)$, there exists $T>0$ such that

$$
\left\|U\left(t+t_{0}, t_{0}, s_{0}\right)-\mathbf{1}_{m} \otimes U_{1}\right\| \leq C_{4} d^{t}
$$

for some $C_{4}>0$, all $t_{0} \geq 0$, and $t \geq T$, where $U_{1}=\left[U_{11}\right.$, $\left.U_{12}, \ldots, U_{1 m}\right]$ the first $n$ rows of $U\left(t+t_{0}, t_{0}, s_{0}\right)$. Then,

$$
\begin{aligned}
& \left\|P^{-1} U\left(t+t_{0}, t_{0}, s_{0}\right) P-P^{-1} \mathbf{1}_{m} \otimes U_{1} P\right\| \\
& =\left\|P^{-1} U\left(t+t_{0}, t_{0}, s_{0}\right) P-\left[\begin{array}{cc}
\gamma(t) & \beta(t) \\
0 & 0
\end{array}\right]\right\| \\
& =\left\|\left[\begin{array}{cc}
0 & \beta(t) \\
0 & \widetilde{U}\left(t+t_{0}, t_{0}, s_{0}\right)
\end{array}\right]\right\| \leq C_{5} d^{t}
\end{aligned}
$$

for some $C_{5}>0$, all $t_{0} \geq 0$, and $t \geq T$, where $\gamma(t)=$ $P_{0}^{\top} \breve{U}\left(t, t_{0}, s_{0}\right) P_{0} \in \mathbb{R}^{n, n}$ and $\beta(t) \in \mathbb{R}^{n, n(m-1)}$ denotes a matrix, and we omit its accurate expression. This implies that $\| \widetilde{U}(t+$ $\left.t_{0}, t_{0}, s_{0}\right) \| \leq C_{6} d^{t}$ holds for some $C_{6}>0$, all $t_{0} \geq 0$, and $t \geq T$. Therefore, we can conclude that $\rho_{P}\left(D \mathscr{F}, s_{0}\right) \leq d$. So, $\rho_{P}\left(D \mathscr{F}, s_{0}\right)=\operatorname{diam}\left(D \mathscr{F}, s_{0}\right)$.

Second, it is clear that $\log \rho_{P}\left(D \mathscr{F}, s_{0}\right) \geq \lambda_{P}\left(D \mathscr{F}, s_{0}\right)$. We will prove that $\log \rho_{P}\left(D \mathscr{F}, s_{0}\right)=\lambda_{P}\left(D \mathscr{F}, s_{0}\right)$. Otherwise, there exists some $r, r_{0}>0$ satisfying $\rho_{P}\left(D \mathscr{F}, s_{0}\right)>r>r_{0}>$ $e^{\lambda_{P}\left(D \mathscr{F}, s_{0}\right)}$. If so, there exists a sequence $t_{k} \uparrow \infty$ as $k \rightarrow$ $\infty, t_{0}^{k} \geq 0$, and $v_{k} \in \mathbb{R}^{n(m-1)}$ with $\left\|v_{k}\right\|=1$ such that $\left\|\widetilde{U}\left(t_{k}+t_{0}^{k}, t_{0}^{k}, s_{0}\right) v_{k}\right\|>r^{t_{k}}$ for all $k \in \mathcal{N}$. Then, there exists a subsequence $v_{k_{l}}$ with $\lim _{l \rightarrow \infty} v_{k_{l}}=v^{*}$. Let $\left\{e_{1}, e_{2}, \ldots, e_{n(m-1)}\right\}$ be a normalized orthogonal basis of $\mathbb{R}^{n(m-1)}$. And, let $v_{k_{l}}-v^{*}=$ $\sum_{j=1}^{n(m-1)} \xi_{j}^{k_{l}} e_{j}$. We have $\lim _{l \rightarrow \infty} \xi_{j}^{k_{l}}=0$ for all $j=1, \ldots, n(m-$ $1)$. Thus, there exists $L>0$ such that

$$
\begin{aligned}
& \left\|\widetilde{U}\left(t_{k_{l}}+t_{0}^{k_{l}}, t_{0}^{k_{l}}, s_{0}\right) v^{*}\right\| \\
& \geq\left\|\widetilde{U}\left(t_{k_{l}}+t_{0}^{k_{l}}, t_{0}^{k_{l}}, s_{0}\right) v_{k_{l}}\right\| \\
& \quad-\left\|\widetilde{U}\left(t_{k_{l}}+t_{0}^{k_{l}}, t_{0}^{k_{l}}, s_{0}\right)\left(v_{k_{l}}-v^{*}\right)\right\| \\
& \geq r^{t_{k_{l}}}-\sum_{j=1}^{n(m-1)}\left|\xi_{j}^{k_{l}}\right|\left\|\widetilde{U}\left(t_{k_{l}}+t_{0}^{k_{l}}, t_{0}^{k_{l}}, s_{0}\right) e_{j}\right\| \\
& \geq r^{t_{k_{l}}}-r_{0}^{t_{k_{l}}}>r_{0}^{t_{k_{l}}}
\end{aligned}
$$

for all $l \geq L$. This implies $e^{\lambda\left(D_{P} \mathscr{F}, v^{*}, s_{0}\right)} \geq r_{0}$ which contradicts with $e^{\lambda_{P}\left(D \mathscr{F}, s_{0}\right)}<r_{0}$. This implies $\rho_{P}\left(D \mathscr{F}, s_{0}\right)=e^{\lambda_{P}\left(D \mathscr{F}, s_{0}\right)}$. Therefore, we can conclude $\log \operatorname{diam}\left(D \mathscr{F}, s_{0}\right)=\lambda_{P}\left(\mathscr{F}, s_{0}\right)$. The proof is completed.

Proof of Lemma 16. Let $\widetilde{\phi}=P^{-1} \phi$. We have

$$
\begin{aligned}
\dot{\tilde{\phi}} & =P^{-1} D F\left(s(t), \varrho^{(t)} \omega_{0}\right) P \tilde{\phi} \\
& =\left[\begin{array}{cc}
P_{0}^{\top} \frac{\partial f}{\partial s}(s(t)) P_{0} & \alpha(t) \\
0 & \widetilde{D} F\left(s(t), \varrho^{(t)} \omega_{0}\right)
\end{array}\right] \tilde{\phi}
\end{aligned}
$$

Write $\widetilde{\phi}=\left[\begin{array}{l}y(t) \\ z(t)\end{array}\right]$, where $y(t) \in \mathbb{R}^{n}$. Then, we have

$$
\begin{gathered}
\dot{z}(t)=\widetilde{D} F\left(s(t), \varrho^{(t)} \omega_{0}\right) z(t), \\
\dot{y}(t)=P_{0}^{\top} \frac{\partial f}{\partial s}(s(t)) P_{0} y(t)+\alpha(t) z(t) .
\end{gathered}
$$

Thus, we can write its solution by

$$
\begin{gathered}
z(t)=\widetilde{U}(t) z_{0}, \\
y(t)=P_{0}^{\top} \breve{U}(t) P_{0} y_{0}+\int_{0}^{t} P_{0}^{\top} \breve{U}(t) \breve{U}^{-1}(\tau) P_{0} \alpha(\tau) \widetilde{U}(\tau) z_{0} d \tau .
\end{gathered}
$$

We write $\lambda_{P}\left(D \mathscr{F}, s_{0}, \omega_{0}\right), \lambda_{S}\left(D \mathscr{F}, s_{0}, \omega_{0}\right)$, and $\lambda_{T}(D \mathscr{F}$, $\left.s_{0}, \omega_{0}\right)$ by $\lambda_{P}, \lambda_{S}$, and $\lambda_{T}$, respectively for simplicity.

Case $1\left(\lambda_{P}>\lambda_{S}\right)$. We can conclude that $\chi[z(t)] \leq \lambda_{P}$ and

$$
\begin{array}{r}
\chi[y(t)] \leq \max \left\{\chi\left[P_{0}^{\top} \breve{U}(t) P_{0} y_{0}\right],\right. \\
\chi\left[\int_{0}^{t} P_{0}^{\top} \breve{U}(t) \breve{U}^{-1}(\tau) P_{0} \alpha(\tau)\right. \\
\times \widetilde{U}(\tau) z(0) d \tau]\} .
\end{array}
$$


From Cauchy-Buniakowski-Schwarz inequality, we have

$$
\begin{gathered}
\chi\left[\left\|\int_{0}^{t} P_{0}^{\top} \breve{U}(t) \breve{U}^{-1}(\tau) P_{0} \alpha(\tau) \widetilde{U}(\tau) d \tau\right\|\right] \\
\leq \chi\left[\left\{\int_{0}^{t}\left\|\breve{U}(t) \breve{U}^{-1}(\tau)\right\|^{2} d \tau\right\}^{1 / 2}\right] \\
+\chi\left[\left\{\int_{0}^{t}\|\alpha(\tau) \widetilde{U}(\tau)\| d \tau\right\}^{1 / 2}\right] .
\end{gathered}
$$

Claim $1\left(\chi\left(\int_{0}^{t}\left\|\breve{U}(t) \breve{U}^{-1}(\tau)\right\|^{2} d \tau\right) \leq 0\right)$. Considering the linear system

$$
\dot{u}(t)=\frac{\partial f}{\partial s}(s(t)) u(t)
$$

due to its regularity and the boundedness of its coefficients, there exists a Lyapunov transform $L(t)$ such that letting $u(t)=L(t) v(t)$, consider the transformed linear system

$$
\begin{aligned}
\dot{v}(t) & =\left[L^{-1}(t) \frac{\partial f}{\partial s}(s(t)) L(t)-L^{-1}(t) \dot{L}(t)\right] v(t) \\
& =\breve{A}(t) v(t) .
\end{aligned}
$$

Let solution matrix $\breve{V}(t)=\left(\breve{v}_{i j}(t)\right)_{i, j=1}^{n}, \breve{A}(t)=\left(\breve{a}_{i j}(t)\right)_{i, j=1}^{n}$ which satisfies that $\breve{A}(t)$ and $\breve{V}(t)$ are lowertriangular. And its Lyapunov exponents can be written as follows:

$$
\sigma_{i}=\lim _{t \rightarrow \infty} \frac{1}{t} \int_{0}^{t} \breve{a}_{i i}(\tau) d \tau,
$$

which are just the Lyapunov exponents of the regular linear $\operatorname{system}(\mathrm{A} .18), i=1,2, \ldots, n$. We have $\chi\left[\breve{v}_{i i}(t)\right]=\sigma_{i}$ and

$$
\begin{aligned}
& \breve{v}_{k+1, k}(t) \\
& \quad=e^{\int_{0}^{t} \breve{a}_{k+1, k+1}(\tau) d \tau} \int_{0}^{t} e^{-\int_{0}^{\tau} \breve{a}_{k+1, k+1}(9) d 9} \breve{a}_{k+1, k}(\tau) \breve{v}_{k, k}(\tau) d \tau .
\end{aligned}
$$

This implies

$$
\chi\left[\breve{v}_{k+1, k}(t)\right] \leq \sigma_{k+1}-\sigma_{k+1}+0+\sigma_{k}=\sigma_{k} .
$$

By induction, we can conclude that $\chi\left[\breve{v}_{j k}(t)\right] \leq \sigma_{k}$ for all $j>$ $k$. For $j<k, \chi\left[\breve{v}_{j k}(t)\right]=-\infty$ due to the lower-triangularity of the matrix $\breve{V}(t)$.

Considering the lower-triangular matrix $\breve{V}^{-1}(t)=$ $\left(\breve{w}_{i j}\right)_{i, j=1}^{n}$, its transpose $\left(\breve{V}^{-1}(t)\right)^{\top}$ can be regarded as the solution matrix of the adjoint system of (A.18):

$$
\dot{w}(t)=-\breve{A}^{\top}(t) w(t),
$$

which is also regular. By the same arguments, we can conclude that $\chi\left[\breve{w}_{k k}\right]=-\sigma_{k}$ for all $k=1,2, \ldots, n, \chi\left[\breve{w}_{j k}\right] \leq-\sigma_{k}$ for all $k>j$, and $\chi\left[\breve{w}_{j k}\right]=-\infty$ for all $k<j$. Therefore, for each $i>j$,

$$
\begin{aligned}
& \max _{i, j} \chi {\left[\int_{0}^{t}\left|\breve{U}(t) \breve{U}^{-1}(\tau)\right|_{i j} d \tau\right] } \\
& \leq \max _{i, j} \chi\left[\int_{0}^{t}\left|\breve{V}(t) \breve{V}^{-1}(\tau)\right|_{i j} d \tau\right] \\
& \leq \max _{i, j} \chi\left[\int_{0}^{t} \sum_{j \leq k \leq i}\left|\breve{v}_{i k}(t) \breve{w}_{k j}(\tau)\right| d \tau\right] \\
& \leq \max _{i, j} \max _{j \leq k \leq i} \chi\left[\int_{0}^{t}\left|\breve{v}_{i k}(t) \breve{w}_{k j}(\tau)\right| d \tau\right] \\
& \leq \max _{i, j} \max _{j \leq k \leq i}\left(\sigma_{k}-\sigma_{k}\right)=0 .
\end{aligned}
$$

This implies that $\chi\left[\int_{0}^{t}\left\|\breve{U}(t) \breve{U}^{-1}(\tau) d \tau\right\|^{2} d \tau\right] \leq 0$.

Noting that

$$
\chi\left[\int_{0}^{t}\|\alpha(\tau) \widetilde{U}(\tau)\|_{2}^{2} d \tau\right] \leq \chi\left[\|\alpha(t) \widetilde{U}(t)\|^{2}\right] \leq 2 \lambda_{P} .
$$

So, $\chi[y(t)] \leq \max \left\{\lambda_{S}, \lambda_{P}\right\}=\lambda_{P}$. This leads to $\chi[\tilde{\phi}(t)] \leq \lambda_{P}$. This implies that $\lambda_{P}=\max \left\{\lambda_{S}, \lambda_{T}\right\}$. Thus, $\lambda_{P}=\lambda_{T}$ can be concluded due to $\lambda_{P}>\lambda_{S}$.

Case $2\left(\lambda_{P}<\lambda_{S}\right)$. For any $\epsilon$ with $0<\epsilon<\left(\lambda_{S}-\lambda_{P}\right) / 3$, there exists $T>0$ such that

$$
\begin{aligned}
& \left\|\breve{U}^{-1}(\tau)\right\| \leq e^{\left(-\lambda_{S}+\epsilon\right) \tau}, \quad\|\alpha(\tau)\| \leq e^{\epsilon \tau}, \\
& \|\widetilde{U}(\tau)\| \leq e^{\left(\lambda_{P}+\varepsilon\right) \tau}
\end{aligned}
$$

for all $t \geq T$. Define the subspace of $\mathbb{R}^{n m}$ :

$$
V=\left\{\left[\begin{array}{l}
y \\
z
\end{array}\right]: y=-\int_{0}^{\infty} P_{0}^{\top} \breve{U}^{-1}(\tau) P_{0} \alpha(\tau) \widetilde{U}(\tau) d \tau z\right\},
$$

which is well defined due to $\left\|P_{0}^{\top} \breve{U}^{-1}(\tau) P_{0} \alpha(\tau) \widetilde{U}(\tau)\right\| \leq$ $e^{\left(3 \epsilon-\lambda_{S}+\lambda_{P}\right) \tau} \in L([T,+\infty))$. For each $\widetilde{\phi}(t)$ with initial condition $\left[\begin{array}{l}y \\ z\end{array}\right] \in V$, we have $\chi[z(t)] \leq \lambda_{P}$ and

$$
\begin{aligned}
& \chi[y(t)] \\
& =\chi\left[\left\{-P_{0}^{-1} \breve{U}(t) P_{0} \int_{0}^{\infty} P_{0}^{\top} \breve{U}^{-1}(\tau) P_{0} \alpha(\tau) \widetilde{U}(\tau) d \tau\right.\right. \\
& \left.\left.+P_{0}^{\top} \breve{U}(t) P_{0} \int_{0}^{t} P_{0}^{\top} \breve{U}^{-1}(\tau) P_{0} \alpha(\tau) \widetilde{U}(\tau) d \tau\right\} z\right] \\
& =\chi\left[-P_{0}^{\top} \breve{U}(t) \int_{t}^{\infty} \breve{U}^{-1}(\tau) P_{0} \alpha(\tau) \widetilde{U}(\tau) d \tau z\right] \leq \lambda_{P}
\end{aligned}
$$

according to the arguments above. Thus, we have $\max _{u \in V} \lambda\left(D \mathscr{F}, u, s_{0}, \omega_{0}\right)=\lambda_{P}$. Since $\operatorname{dim}(V)=n(m-1), V$ define the transverse space and $\lambda_{T}=\lambda_{P}$. This completes the proof. 
Proof of Lemma 22. Since $L(t)$ satisfies Assumption 20, if the initial condition is $u\left(t_{0}\right)=\mathbf{1}_{m}$, then the solution must be $u(t)=\mathbf{1}_{m}$, which implies that each row sum of $V\left(t, t_{0}\right)$ is one. Then, we will prove all elements in $V\left(t, t_{0}\right)$ are nonnegative. Consider the $i$ th column of $V\left(t, t_{0}\right)$ denoted by $V^{i}\left(t, t_{0}\right)$ which can be regarded as the solution of the following equation:

$$
\begin{gathered}
\dot{u}=\sigma L(t) u, \\
u\left(t_{0}\right)=e_{i}^{m} .
\end{gathered}
$$

For any $t \geq t_{0}$, if $i_{0}=i_{0}(t)$ is the index with $u_{i_{0}}(t)=$ $\min _{i=1,2, \ldots, m} u_{i}(t)$, we have $\dot{u}_{i_{0}}(t)=\sum_{j=1}^{m} \sigma l_{i_{0} j}\left(u_{j}(t)-u_{i_{0}}(t)\right) \geq 0$. This implies that $\min _{i=1,2, \ldots, m} u_{i}(t)$ is always nondecreasing for all $t \geq t_{0}$. Therefore, $u_{i}(t) \geq 0$ holds for all $i=1,2, \ldots, m$ and $t \geq t_{0}$. We can conclude that $V\left(t, t_{0}\right)$ is a stochastic matrix. The proof is completed.

Proof of Lemma 29. Consider the following Cauchy problem:

$$
\begin{gathered}
\dot{u}_{i}(t)=\sum_{j=1}^{m} \sigma l_{i j}(t) u_{j}(t), \\
u_{i}\left(t_{0}\right)= \begin{cases}1, & i=k \\
0, & \text { otherwise }\end{cases} \\
i=1,2, \ldots, m .
\end{gathered}
$$

Noting that $\dot{u}_{k}(t) \geq \sigma l_{k k} u_{k}$, we have $u_{k}(t) \geq e^{-M_{1}\left(t-t_{0}\right)}$. For each $i \neq k$, since $u_{i}(t) \geq 0$ for all $i=1,2, \ldots, m$ and $t \geq t_{0}$, we have

$$
\begin{aligned}
u_{i}(t) & =\sum_{j \neq i} \int_{t_{0}}^{t} e^{\int_{\tau}^{t} \sigma l_{i i}(\vartheta) d \vartheta} \sigma l_{i j}(\tau) u_{j}(\tau) d \tau \\
& \geq \int_{t_{0}}^{t} e^{\int_{\tau}^{t} \sigma l_{i i}(9) d \vartheta} \sigma l_{i k}(\tau) u_{k}(\tau) d \tau \\
& \geq \int_{i_{0}}^{t} e^{-M_{1}(t-\tau)} e^{-M_{1}\left(\tau-t_{0}\right)} \sigma l_{i k}(\tau) d \tau \\
& =e^{-M_{1}\left(t-t_{0}\right)} \int_{t_{0}}^{t} \sigma l_{i k}(\tau) d \tau
\end{aligned}
$$

So, if there exists a $\delta$-edge from vertex $j$ to $i$ across $\left[t_{0}\right.$, $\left.t_{0}+T\right]$, then we have $v_{i j}\left(t_{0}+T, t_{0}\right) \geq e^{-M_{1} T} \delta$. Let $\delta_{2}=\min \left\{e^{-M_{1} T}, e^{-M_{1} T} \delta\right\}$. We can see that $V\left(t, t_{0}\right)$ has a $\delta_{2}$ spanning tree across any $T$-length time interval. Therefore, according to $[31,32]$, there exist $\delta_{1}>0$ and $T_{1}=(m-1) T$ such that $V\left(t, t_{0}\right)$ is $\delta_{1}$ scrambling across any $T_{1}$-length time interval. The Lemma is proved.

\section{Acknowledgments}

This work is jointly supported by the National Key Basic Research and Development Program (no. 2010CB731403), the National Natural Sciences Foundation of China under Grant (nos. 61273211 and 61273309), the Shanghai Rising-Star Program (no. 11QA1400400), the Marie Curie International
Incoming Fellowship from the European Commission under Grant FP7-PEOPLE-2011-IIF-302421, and the Laboratory of Mathematics for Nonlinear Science, Fudan University.

\section{References}

[1] M. Dhamala, V. K. Jirsa, and M. Ding, "Transitions to synchrony in coupled bursting neurons," Physical Review Letters, vol. 92, no. 2, pp. 281011-281014, 2004.

[2] V. Perez-Munuzuri, V. Perez-Villar, and L. O. Chua, "Autowaves for image processing on a two-dimensional CNN array of excitable nonlinear circuits: flat and wrinkled labyrinths," IEEE Transactions on Circuits and Systems I, vol. 40, no. 3, pp. 174-181, 1993.

[3] P. Ashwin, J. Buescu, and I. Stewart, "From attractor to chaotic saddle: a tale of transverse instability," Nonlinearity, vol. 9, no. 3, pp. 703-737, 1996.

[4] J. Milnor, "Correction and remarks: 'On the concept of attractor," Communications in Mathematical Physics, vol. 102, no. 3, pp. 517-519, 1985.

[5] J. Milnor, "On the concept of attractor," Communications in Mathematical Physics, vol. 99, no. 2, pp. 177-195, 1985.

[6] W. Lu, F. M. Atay, and J. Jost, "Synchronization of discrete-time dynamical networks with time-varying couplings," SIAM Journal on Mathematical Analysis, vol. 39, no. 4, pp. 1231-1259, 2007/08.

[7] A. Margheri and R. Martins, "Generalized synchronization in linearly coupled time periodic systems," Journal of Differential Equations, vol. 249, no. 12, pp. 3215-3232, 2010.

[8] J. Lu, D. W. C. Ho, J. Cao, and J. Kurths, "Exponential synchronization of linearly coupled neural networks with impulsive disturbances," IEEE Transactions on Neural Networks, vol. 22, no. 2, pp. 329-335, 2011.

[9] C. W. Wu, "Synchronization in networks of nonlinear dynamical systems coupled via a directed graph," Nonlinearity, vol. 18, no. 3, pp. 1057-1064, 2005.

[10] V. N. Belykh, I. V. Belykh, and M. Hasler, "Connection graph stability method for synchronized coupled chaotic systems," Physica D, vol. 195, no. 1-2, pp. 159-187, 2004.

[11] I. V. Belykh, V. N. Belykh, and M. Hasler, "Blinking model and synchronization in small-world networks with a time-varying coupling," Physica D, vol. 195, no. 1-2, pp. 188-206, 2004.

[12] J. Lü and G. Chen, "A time-varying complex dynamical network model and its controlled synchronization criterion," IEEE Transactions on Automatic Control, vol. 50, no. 2, pp. 169-182, 2005.

[13] W. L. Lu, F. M. Atay, and J. Jost, "Chaos synchronization in networks of coupled maps with time-varying topologies," The European Physical Journal B, vol. 63, no. 3, pp. 399-406, 2008.

[14] B. Shen, Z. Wang, and X. Liu, "Sampled-data synchronization control of dynamical networks with stochastic sampling," IEEE Transactions on Automatic Control, vol. 57, no. 10, pp. 26442650, 2012.

[15] B. Shen, Z. Wang, and X. Liu, "Bounded $H_{\infty}$ synchronization and state estimation for discrete time-varying stochastic complex networks over a finite horizon," IEEE Transactions on Neural Networks, vol. 22, no. 1, pp. 145-157, 2011.

[16] D. J. Stilwell, E. M. Bollt, and D. G. Roberson, "Sufficient conditions for fast switching synchronization in time-varying network topologies," SIAM Journal on Applied Dynamical Systems, vol. 5, no. 1, pp. 140-156, 2006. 
[17] W. Lu, T. Chen, and G. Chen, "Synchronization analysis of linearly coupled systems described by differential equations with a coupling delay," Physica D, vol. 221, no. 2, pp. 118-134, 2006.

[18] W. Lu and T. Chen, "New approach to synchronization analysis of linearly coupled ordinary differential systems," Physica D, vol. 213, no. 2, pp. 214-230, 2006.

[19] B. Liu, W. Lu, and T. Chen, "New conditions on synchronization of networks of linearly coupled dynamical systems with nonLipschitz right-hand sides," Neural Networks, vol. 25, pp. 5-13, 2012.

[20] J. K. Hale, Ordinary Differential Equations, Wiley-Interscience, New York, NY, USA, 1969.

[21] L. Ya. Adrianova, Introduction to Linear Systems of Differential Equations, vol. 146 of Translations of Mathematical Monographs, American Mathematical Society, Providence, RI, USA, 1995.

[22] B. Liu, W. Lu, and T. Chen, "Consensus in networks of multiagents with switching topologies modeled as adapted stochastic processes," SIAM Journal on Control and Optimization, vol. 49, no. 1, pp. 227-253, 2011.

[23] J. Hajnal, "Weak ergodicity in non-homogeneous Markov chains," Proceedings of the Cambridge Philosophical Society, vol. 54, pp. 233-246, 1958.

[24] J. Hajnal, "The ergodic properties of non-homogeneous finite Markov chains," Proceedings of the Cambridge Philosophical Society, vol. 52, pp. 67-77, 1956.

[25] L. Moreau, "Stability of continuous-time distributed consensus algorithms," in Proceedings of the 43rd IEEE Conference on Decision and Control (CDC '04), vol. 4, pp. 3998-4003, December 2004.

[26] O. E. Rössler, "Continuous chaos four prototype equations," Annals of the New York Academy of Sciences, vol. 316, pp. 376392, 1979.

[27] M. E. J. Newman and D. J. Watts, "Scaling and percolation in the small world network model," Physical Review E, vol. 60, no. 6, pp. 7332-7342, 1999.

[28] A. Wolf, J. B. Swift, H. L. Swinney, and J. A. Vastano, "Determining Lyapunov exponents from a time series," Physica D, vol. 16, no. 3, pp. 285-317, 1985.

[29] G. Barna and I. Tsuda, "A new method for computing Lyapunov exponents," Physics Letters A, vol. 175, no. 6, pp. 421-427, 1993.

[30] M. Sandri, "Numerical calculation of Lyapunov exponents," Mathematica Journal, vol. 6, no. 3, pp. 78-84, 1996.

[31] J. Wolfowitz, "Products of indecomposable, aperiodic, stochastic matrices," Proceedings of the American Mathematical Society, vol. 14, no. 5, pp. 733-737, 1963.

[32] V. I. Oseledec, "A multiplicative ergodic theorem. Characteristic Ljapunov, exponents of dynamical systems," Transactions of the Moscow Mathematical Society, vol. 19, pp. 197-231, 1968. 


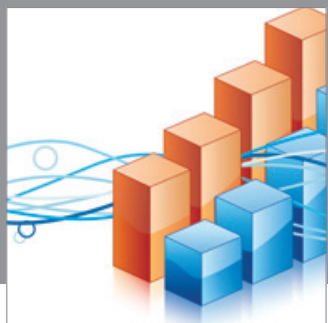

Advances in

Operations Research

mansans

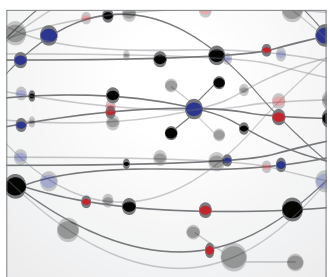

The Scientific World Journal
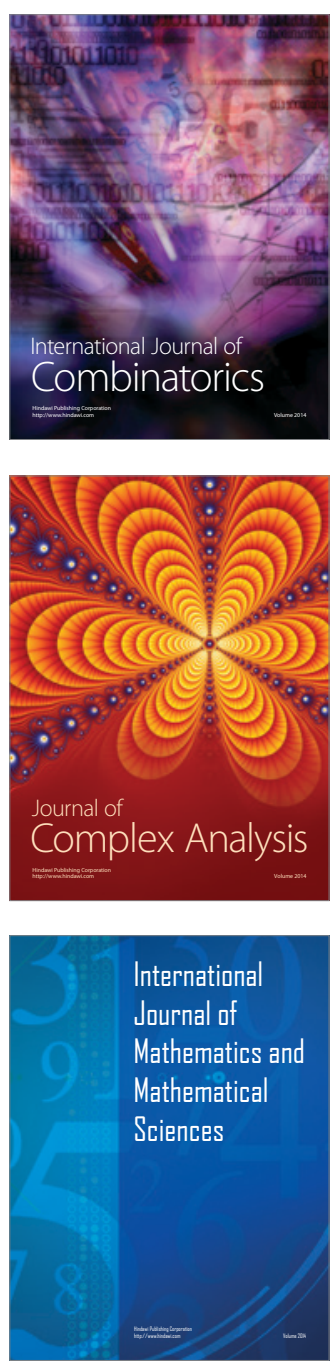
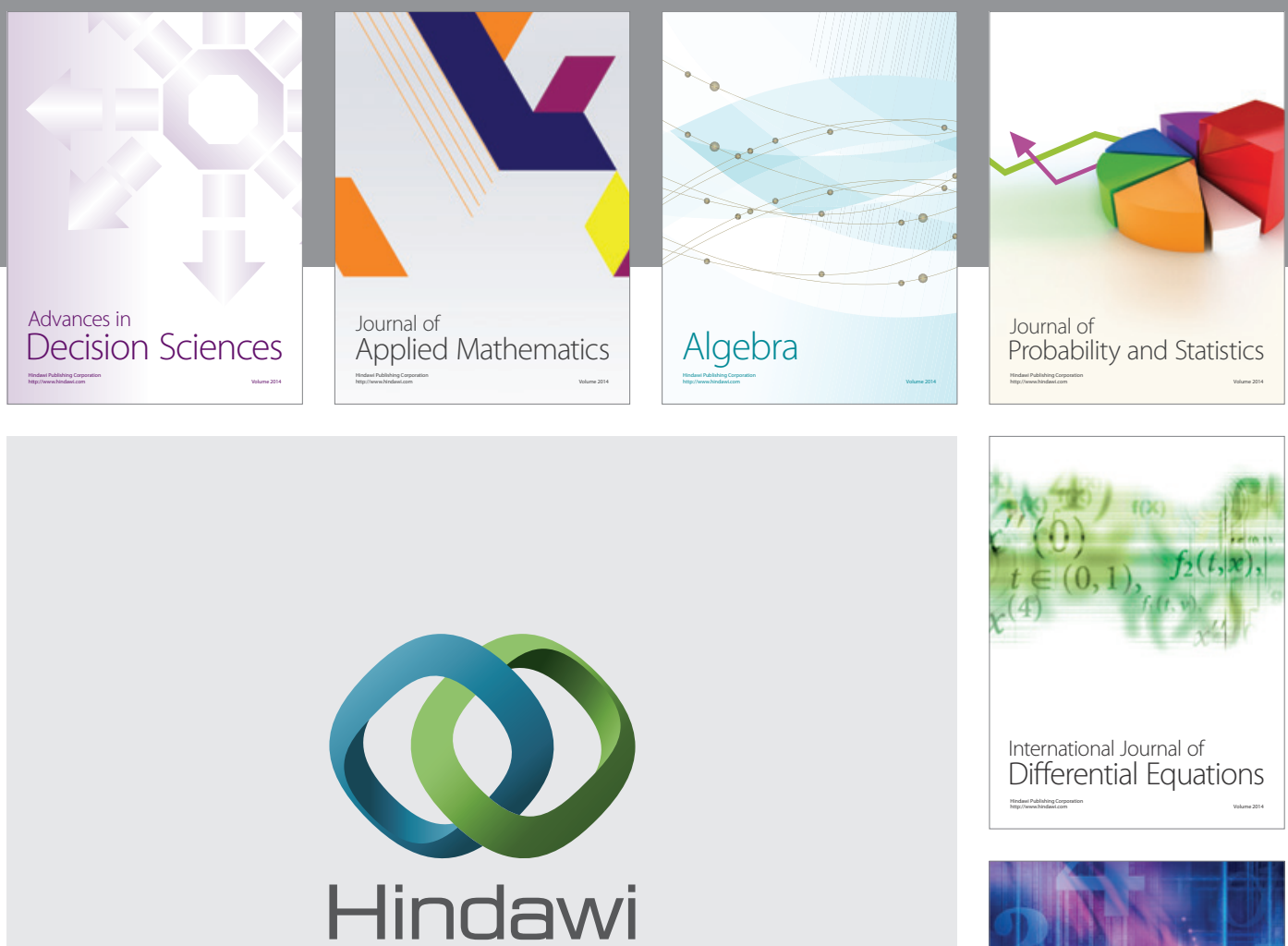

Submit your manuscripts at http://www.hindawi.com
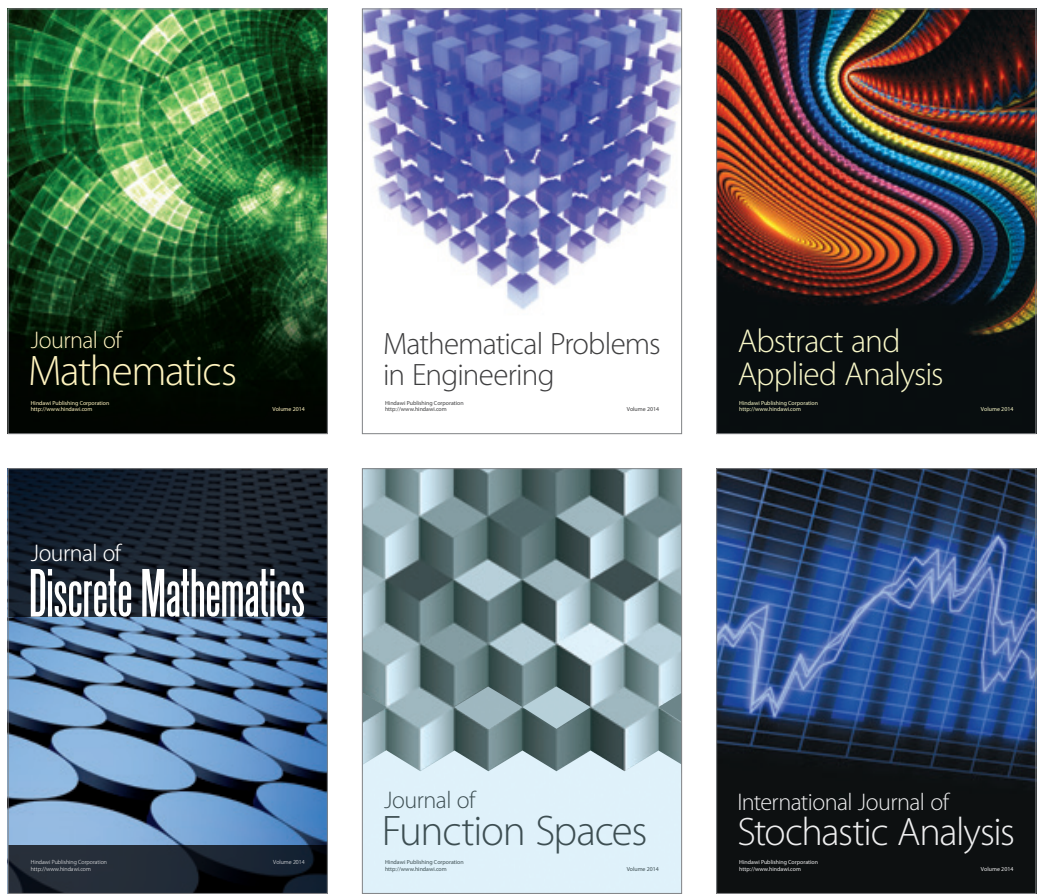

Journal of

Function Spaces

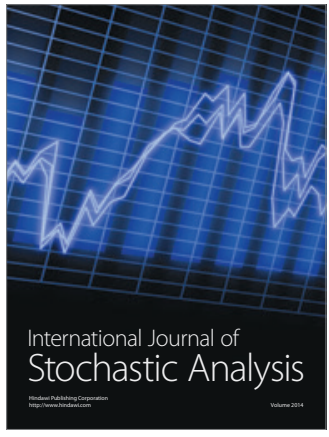

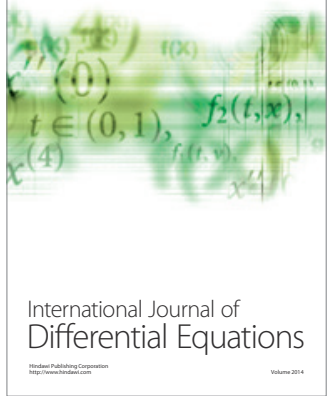
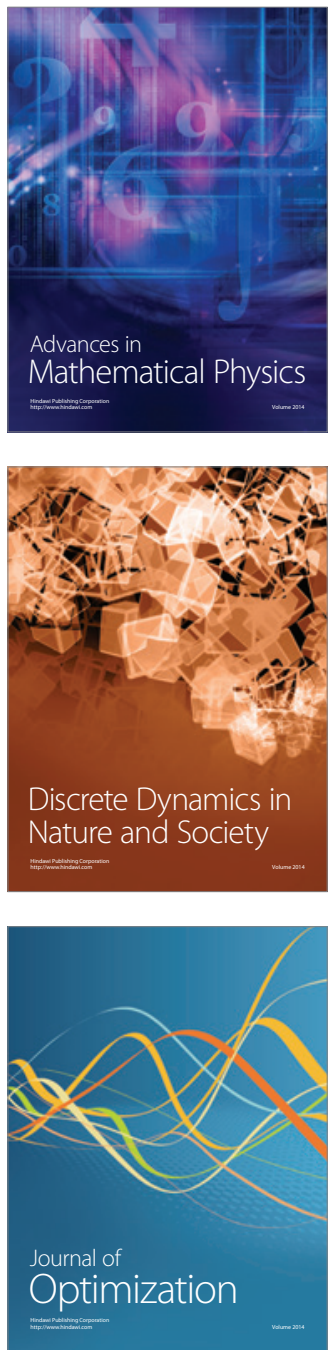